\title{
Controling switching between birhythmic states in a new conductance-based bursting neuronal model
}

\author{
I. Tagne Nkounga, ${ }^{1}$ F. M. Moukam Kakmeni, ${ }^{2}$ B. I. Camara,${ }^{3}$ and R. Yamapi $^{1}$, , \\ ${ }^{1}$ Fundamental Physics Laboratory, Physics of Complex System group, \\ Department of Physics, Faculty of Science, \\ University of Douala, Box 24157 Douala, Cameroon \\ ${ }^{2}$ Complex Systems and Theoretical Biology Group and Laboratory of \\ Research on Advanced Materials and Non-linear Science (LaRAMaNS), \\ Department of Physics, Faculty of Science, \\ University of Buea, P.O. Bох 63, Buea, Cameroon \\ ${ }^{3}$ Laboratoire Interdisciplinaire des Environnements Continentaux \\ Université de Lorraine - CNRS UMR 7360 \\ Campus Bridoux - 8 Rue du Général Delestraint, 57070 Metz, France
}

(Dated: September 2, 2021)

\begin{abstract}
A birhythmic conductance-based neuronal model with fast and slow variables is proposed to generate and control the coexistence of two different attracting modes in amplitudes and frequencies. However, periodic bursting, chaotic spiking and bursting haven't been clearly observed there. The control of bistability is investigated in a three-dimensional birhythmic conductance-based neuronal model. We consider slow processes in neuron materialized by an adaptation variable coupled to system in the presence of an external sinusoidal current applied. By using the harmonic balance method, we obtain the frequency-response curve in which membrane potential resonance with his corresponding frequency are control by varying a specific parameter. At the resonance frequency, bifurcation and lyapunov exponent diagrams versus a control parameter are obtained. They reveal, a coexistence of two different complex attractors namely periodic and chaotic spiking, periodic and chaotic bursting. By using the control parameter as the slow variable, the system can switch from bistable to monostable behavior. This is done by destroying subthreshold (small) oscillation of the neuron. The role of adaptation variable in neuron system is then to filtered many existing electrical processes and permit to adapt the system by the multiple transitions states in the chosen electrical mode. A fairly good agreement is observed between analytical and numerical results.
\end{abstract}

Keywords: Birhythmicity, conductance-based neuronal model; membrane potential resonance; periodic and chaotic spiking, bursting oscillations; switching; monostability; control.

PACS numbers:

*ryamapi@yahoo.fr(Corresponding authors) 


\section{INTRODUCTION}

Multistability is a common dynamical feature of many natural systems [1]. It has been observed in modelling studies [2] such as coexistence of several attractors in the phase space of a system. Bistability is an interesting manifestation of multistability which occurs very frequently and deserve special attention due to their importance in physics and biology. It plays a significant role in living systems in maintaining different modes of oscillations to various biochemical processes in varying environment [3]. It's a fundamental attribute of the dynamic of neurons and neuronal networks [4] because neurons can be viewed as strongly non-linear dynamical capable of generating complex patterns of activity. The coexistence of different attracting regimes of activity that reflect their intrinsic ionic channel behaviors within a specific parameter range, is not uncommon for a such system. The particular attractor exhibited by it, is determined by initial conditions. Multistability by bifurcations phenomena sets a remarkable framework for potential dynamical plasticity of neurons with implications for dynamical memory, information processing and motor control [5].

The coexistence of neuronal activity regimes has been observed and demonstrated by theoretical and experimental studies [6] and recently by Tagne Nkounga et al. [7]. One of the most studies coexistence states is the coexistence of tonic spiking and silence [8]-10]. Compared with the Hodgkin Huxley (HH)[11] and the 2D Moris Lecar (ML)[8] models, the 2D birhythmic conductance-based neuronal model is so simple. In fact, this mathematical model can generate various action voltage potentials [7]. It cannot trigger bursting firings or complex dynamical transition from periodic to chaotic motions [12] that appear generally in biological neuronal systems with fast and slow variables. Because the using of multistability can permit to develop therapeutic strategies of some diseases such as epilepsy, it's so important to identify an intrinsic property to control multistability which occur in neuron. Many studies have showed a way to control multistability in living systems. Guttman et al. [13] have shown in experiment on the squid giant axon in saline with low calcium concentration that a switching between two regimes can be executed by a pulse of current. Ghosh et al. [14] reported an effective control mechanism of birhythmic behavior in a modified van der Pol system by using a variant of Pyragas technique of time delay control [15]. They have shown that depending upon the time-delay, system can induce monorhythmic oscillations out of birhythmicity. Ghosh et al. [14] established that their technique can suppress the effective birhythmic zone.

The 3D HR neuronal system have shown that the third linear equation added to their two dimensional model can with a variant parameter, control a rhythmic states transitions of attractors. This exhibited periodic spiking, periodic and chaotic bursting [16]. In the Jerk systems, the control of multistability was based on the linear augmentation scheme [17]. It's also showed in a leech model that the adding of slow variable to fast subsystem, can switch the system from the coexistence states [6]. Altough the coexistence of complex dynamics presented in some biophysical 
models, these models are complex and the demonstration of these phenomena are generally done through numerical simulations. In the present work, we propose and analyse a 3D birhythmic conductance-based neuronal model. The model derived from the 3D prescott model [18], introduced as an extension of the 2D ML model. We focuses on the control of switching between coexisting attractors. The importance of the present work reside on the fact that it possesses multilimit cycles. More importantly, we analytical define the system parameter domain where these many attractors coexist. On the contrary of other models where the coexistence between two periodic regimes (birhythmicity), random oscillations (chaos), and coexistence of a stable limit-cycle oscillations are done numerically [19-21], our model offer a room for analytical analysis. Note that, the understanding of the duality (birhythmicity) of neurons has motived many researchers in proposing some models [17, 19-21]. The faithfulness of these models to underline that biological neuron and it complexity is crucial in describing the information processing and transmission in neuronal system [5]. Thus, by considering slow processes as an adaptation variable in our model and by applying an external sinusoidal current, we analyse analytically and numerically, the coexistence of periodic or/and chaotic regimes and the control of these various activities in the neuron.

The work is organized as follows: In section II, we use a 3D Prescott model to modeling a 3D birhythmic conductance based neuronal model by adding a slow variable than the first two in the 2D model. In section III, we proceed to a deterministic dynamical analysis of the model in absence of a third variable. The amplitudes and frequencies of oscillatory states and their stability are study using respectively the Lindsted's perturbation method and the Floquet theory. We then study the effect of the third added variable without and with a forcing term on a 3D birhythmic conductancebased neuronal model. These using firstly analytical methods as harmonic decomposition and harmonic balance method respectively, and secondly numerical methods as the bifurcation and lyapunov exponent. In section IV, we conclude this work.

\section{THE BIRHYTHMIC CONDUCTANCE-BASED NEURONAL MODEL AND DYNAMICAL} ANALYSIS

\section{A. The conductance-based neuronal model}

Many mathematical models were used to describe neural activity. A two-dimensional Prescott neuron model is an improvement of ML neuron model (describe the oscillations in the barnacle giant muscle fiber) [18]. It consists of a fast activation variable and a slow recovery variable or activation of potassium current [22]. A three-dimensional Prescott model introduces an adaptive ion current due to sodium channel inactivation since it operates on the same time scale as delayed rectified channel activation. This to make the model more realistic (a real description to emission of membrane potential about the long time scale between action potential). Since adaptability is a 


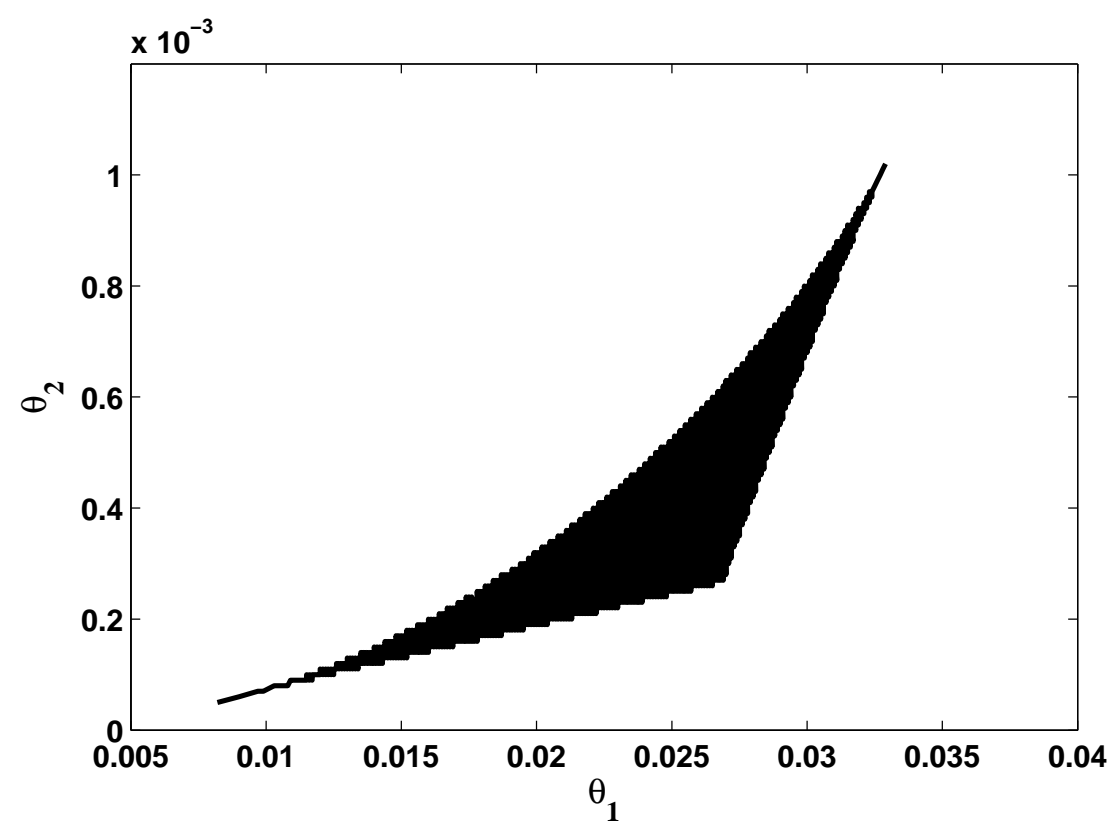

Figure 1: Region of existence to one (unhatched part) and three limit-cycles (hatched part) in the plane ( $\theta_{1}$, $\theta_{2}$ ) obtained by solving the algebraic equation (9).
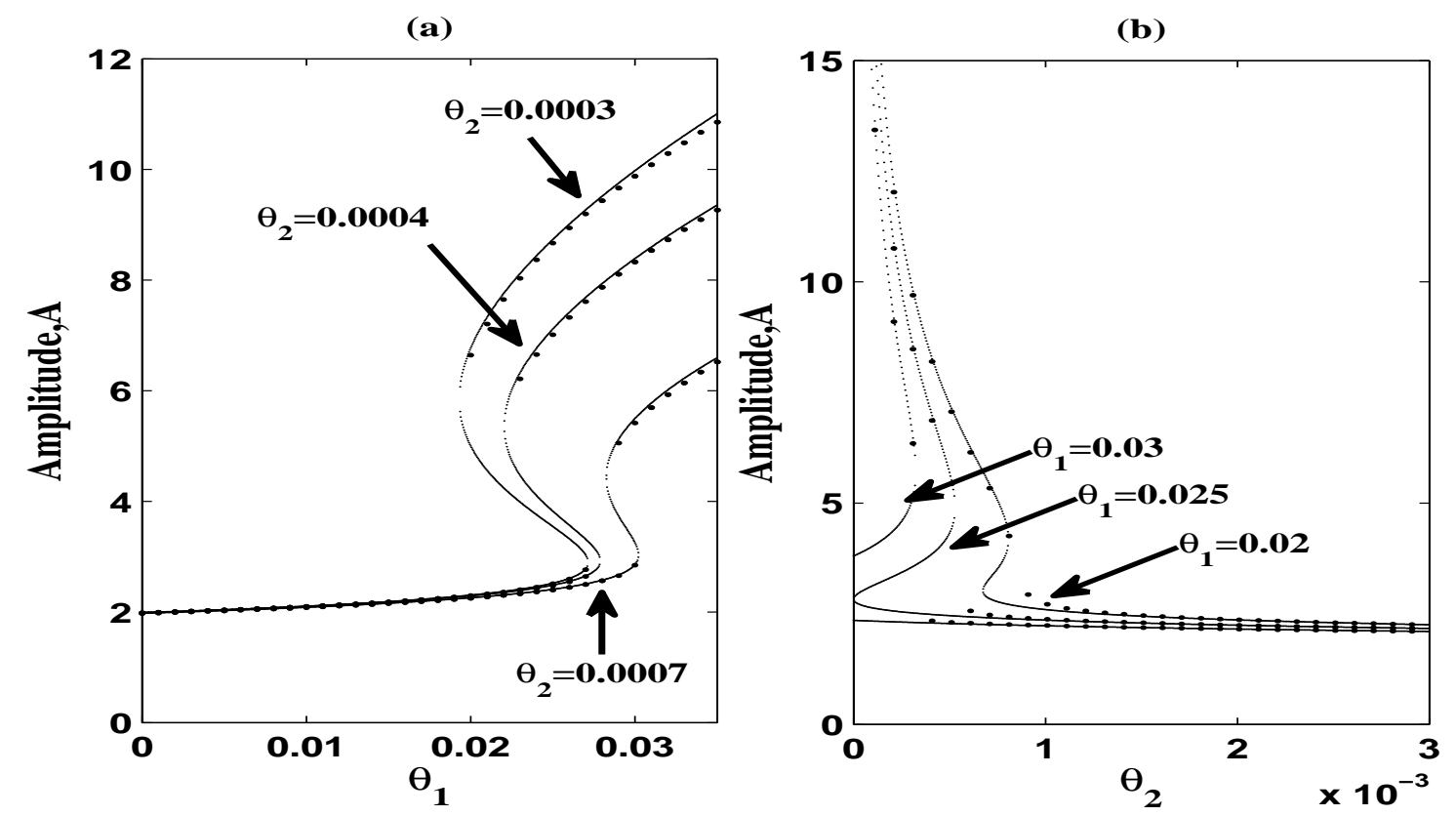

Figure 2: Amplitude-response versus $\theta_{1}$ for several different values of $\theta_{2}(a)$ and versus $\theta_{2}$ for several different values of $\theta_{1}(b)$. The parameter used is: $\gamma_{1}=35.0$ 
(a)

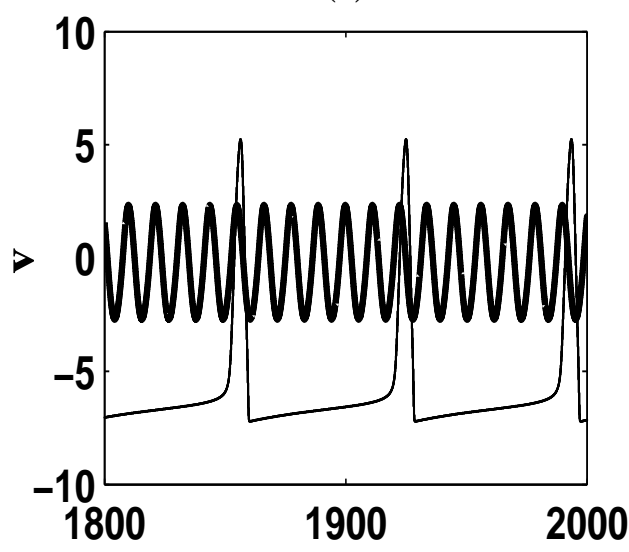

(c)

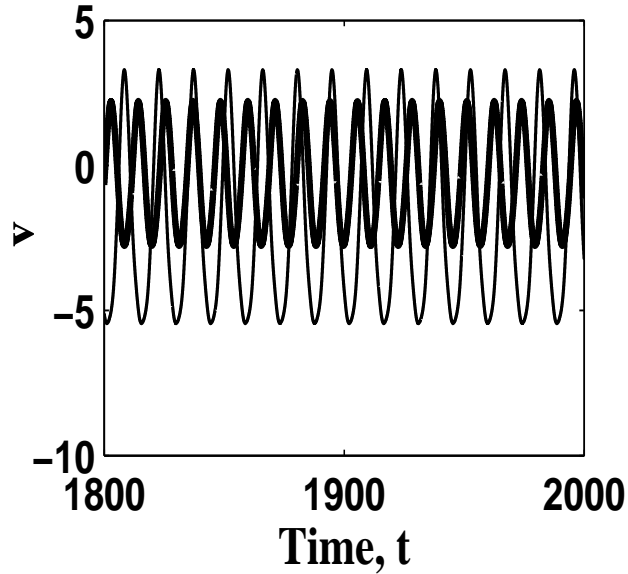

(b)

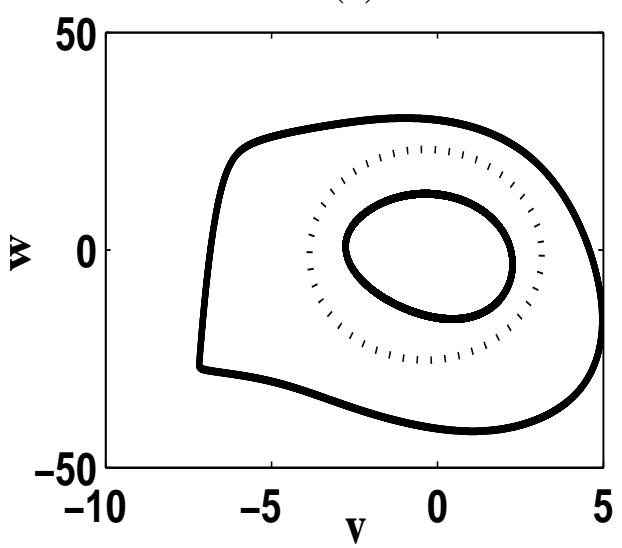

(d)

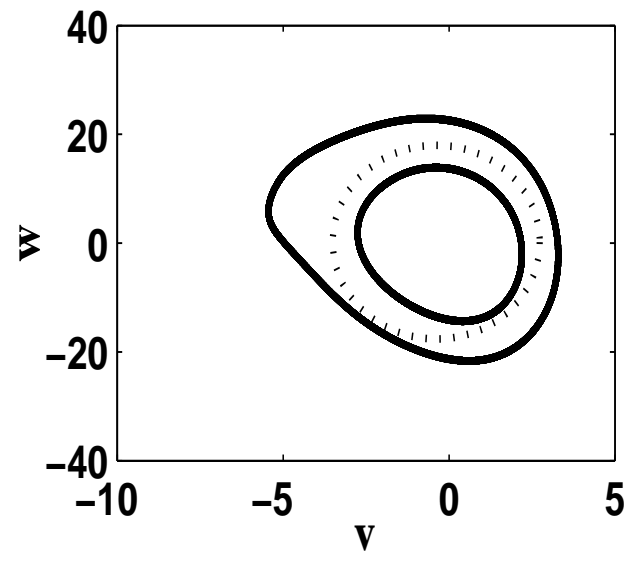

Figure 3: The coexistence between two regimes of self-sustained oscillations corresponding to the smaller (amplitude $A_{1}$, and frequency $\omega_{1}$ ) and larger (amplitude $A_{3}$, and frequency $\omega_{3}$ ) limit cycles. The set of parameters is the same as for the attractors shown in Table I. The parameters used are: $\gamma_{1}=35.0$ and $S_{2}:(a),(b) ; S_{4}:(c),(d)$

prominent characteristic of neural dynamics, the dynamical equations are as follows:

$$
\left\{\begin{array}{l}
\frac{d v}{d t}=I+\frac{1}{C}\left[g_{N a} m_{\infty}(v)(b-w)\left(E_{N a}-v\right)+g_{K} w\left(E_{K}-v\right)+g_{L}\left(E_{L}-v\right)+g_{a} z\left(E_{a}-v\right)\right] \\
\frac{d w}{d t}=\frac{w_{\infty}(v)-w}{\tau_{w}(v)} \\
\frac{d z}{d t}=\frac{z_{\infty}(v)-z}{\tau_{z}(v)}
\end{array}\right.
$$



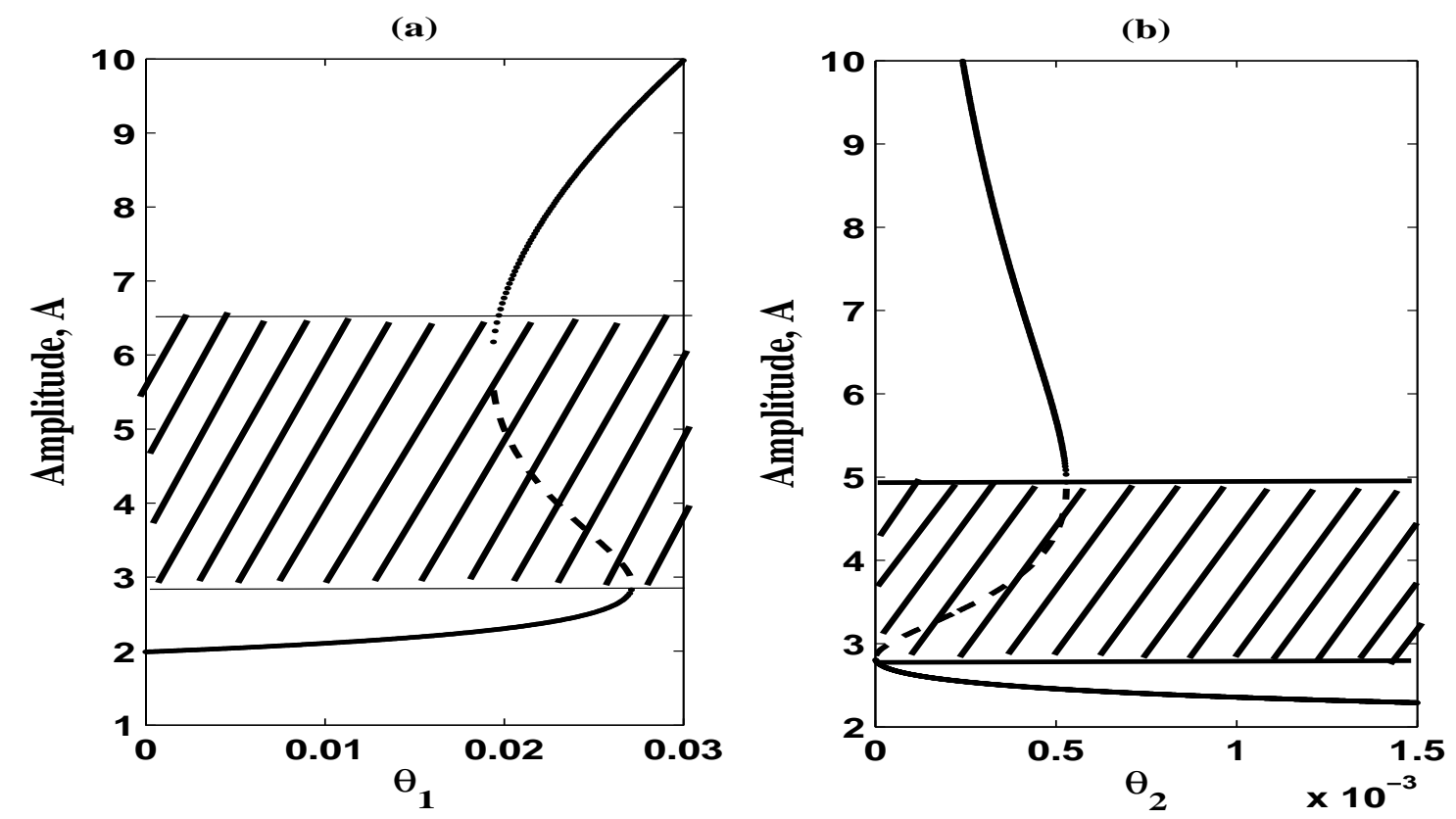

Figure 4: Regions of stability and instability (dotted line) for amplitude versus $\theta_{1}, \theta_{2}=0.0003(a)$ and $\theta_{2}, \theta_{1}=0.025(b)$ The parameters used is: $\gamma_{1}=35.0$

where

$$
\begin{aligned}
& m_{\infty}(v)=\frac{1}{2}+\frac{1}{2} \tanh \left(\frac{v-\beta_{m}}{\gamma_{m}}\right), \\
& w_{\infty}(v)=\frac{1}{2}+\frac{1}{2} \tanh \left(\frac{v-\beta_{w}}{\gamma_{w}}\right), \\
& z_{\infty}(v)=\frac{1}{2}+\frac{1}{2} \tanh \left(\frac{v-\beta_{z}}{\gamma_{z}}\right), \\
& \tau_{w}(v)=\frac{\phi_{w}}{\cosh \left(\frac{v-\beta_{w}}{2 \gamma_{w}}\right)} \\
& \tau_{z}(v)=\frac{\phi_{z}}{\cosh \left(\frac{v-\beta_{z}}{2 \gamma_{z}}\right)} .
\end{aligned}
$$

The system (1) consists of voltage-gated $N_{a}^{+}$current, delayed rectified $K^{+}$current, adaptive currents and the leak current. Here $v, w, z$ represent the membrane potential of the neuron, the activation variable of $K^{+}$ion channels (the recovery variable of slow ionic currents), and the gating variable of the adaptive currents respectively. The parameters $g_{N a}, g_{K}, g_{a}$ and $g_{L}$ indicate the maximum conductance functions of ions $N_{a}^{+}, K^{+}$, leak currents and adaptive currents respectively. $E_{N a}, E_{K}, E_{a}$ and $E_{L}$ are reversal potentials of the different tonic current functions. $C$ mean the membrane capacitance and it is considered as unity. $\phi_{w}$ and $\phi_{z}$ represents the temperature scaling 
factor for the $K^{+}$channel opening and ions adaptive currents respectively. $\beta_{m}, \gamma_{m}, \beta_{w}, \gamma_{w}, \beta_{z}$, $\gamma_{z}$ are other parameters of model, $I$ is the external current applied to the model. In the presence of adaptative current, neuron can exhibited periodic and chaotic bursting, coexisting multistable firing and the transition to each other as shows in the 3D ML model [23]. In the present work, the three trigonometric hyperbolic functions present in a system, are Expanding in the taylor series considering the higher-order nonlinear terms and taking into account some selected terms, we can written eq.(1) in the form:

$$
\left\{\begin{array}{l}
\frac{d v}{d t}=B w+a v-b v^{3}+\theta_{1} v^{5}-\theta_{2} v^{7}-\alpha z+I \\
\frac{d w}{d t}=c-\gamma_{1} v-\gamma_{2} v^{2}-\gamma_{3} w \\
\frac{d z}{d t}=\gamma_{4}+r(s v-z)
\end{array}\right.
$$

where, the parameters $a, b, B, \alpha, \theta_{1}, \theta_{2}, c, r, s, \gamma_{1}, \gamma_{2}, \gamma_{3}, \gamma_{4}$ and their range values, are given in appendix . It's also noted as in [7] that $\theta_{1}, \theta_{2}, \alpha$ are the function of the maximum conductance of ions channels. By setting $a=2.0, c=B=\gamma_{3}=1.0, b=1 / 3, \gamma_{4}=0.0, s=4.0$ and $\gamma_{2}=5.0$, we obtain the new neuronal mathematical model below which will be used in all this analysis:

$$
\left\{\begin{array}{l}
\frac{d v}{d t}=2 v-\frac{1}{3} v^{3}+\theta_{1} v^{5}-\theta_{2} v^{7}+w-\alpha z+I=f(v)+w-\alpha z+I \\
\frac{d w}{d t}=1-\gamma_{1} v-5 v^{2}-w=g(v)-w, \\
\frac{d z}{d t}=r(s v-z) .
\end{array}\right.
$$

The model described by eq. (4) is obtained by an approximation of the biological improved MorrisLecar neuron model namely Prescott neuron model describing the neuronal dynamic. Contrary to a simple three dimensional mathematical model as HR where the function $f$ are taken as a cubic polynomial function, we establish this simple model in which $f$ is a septic polynomial function and $g$ is a quadratic polynomial function. In the simple tridimensional model of HR [16] as in the three-dimensional Prescott model [22], the third variable is shows as a variable of bifurcation control of a multistable dynamic. Thus, a brief current given by the variable $z$ called adaptation variable whose phase and amplitude given in an appropriate range, can provides hysteresis and can switches the system between coexistent stable states [24]. It also may suppress bistability or maintain desired dynamical behaviors in some case.

\section{B. Amplitudes, frequencies of oscillatory states and their stability}

In optic to find concordance of analytical and numerical results, we proceed to reduce equations of system (4) in the absence of adaptation variable $z(\alpha=0)$ and with a constant external current, in nonlinear equation that we can solve by non linear analytical analysis methods known by all. 

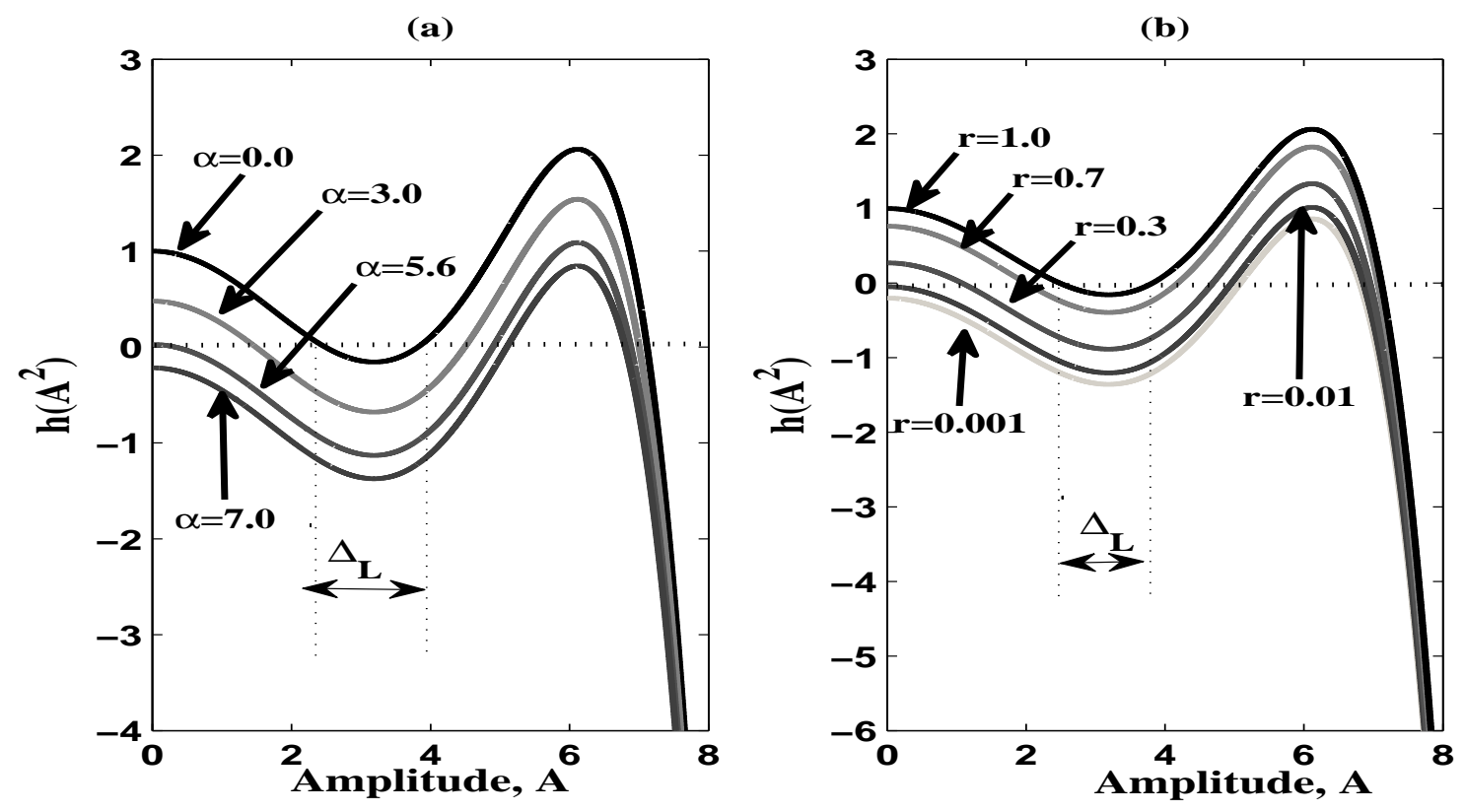

Figure 5: Curves $h\left(A^{2}\right)$ versus A respectively for many values of $\alpha, r=0.0001$ (a) and for many values of $r, \alpha=7.0(b)$. The parameters used are: $\gamma_{1}=35.0$ and $S_{2}:(a),(b)$

That is why, by eliminating $w$ in the system (4), we obtain after setting $t=\omega_{0} \tau$, the following equation similar to what found in [25]:

$$
\ddot{x}-\mu\left(1-x^{2}+\alpha_{1} x^{4}-\alpha_{2} x^{6}\right) \dot{x}+x=\mu^{2}\left(1-5 x^{2}-\frac{1}{3} x^{3}+\frac{1}{5} \alpha_{1} x^{5}-\frac{1}{7} \alpha_{2} x^{7}\right)+I_{e x t},
$$

where,

$$
\begin{array}{rlrl}
\mu & =\frac{1}{\Omega_{0}}, \quad \alpha_{1} & =5 \theta_{1}, \quad \alpha_{2}=7 \theta_{2}, \quad \Omega_{0}=\sqrt{\gamma_{1}-2}, \\
v(t) & =x, \quad I(t)=\frac{\beta_{2}}{\omega_{0}^{2} \sqrt{1-\beta_{2}}} I\left(\frac{t}{\Omega_{0}}\right)=I_{\text {ext }} .
\end{array}
$$

This equation is of the leanard form and can be solve analytically using the Lindsted's perturbation method [26]. In order to found amplitudes and frequencies of the system, we posed $\tau=\omega t$, where $\omega$ is a unkown frequency. The periodic solution of (5) can be express by:

$$
x(\tau)=x_{0}(\tau)+\mu x_{1}(\tau)+\mu^{2} x_{2}(\tau)+\ldots,
$$

where $x_{n}(\tau)(n=0,1,2, \ldots)$ are periodic functions of $\tau$ of period $2 \pi$. therefore, the frequency $\omega$ can be represented by the following expression:

$$
\omega=\omega_{0}+\mu \omega_{1}+\mu^{2} \omega_{2}+\ldots
$$


(a)

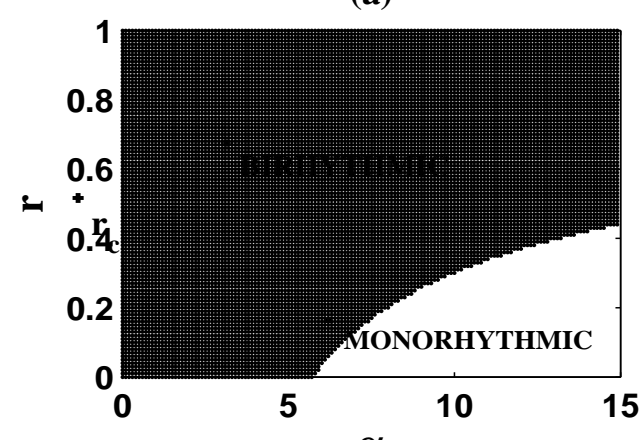

$\alpha$
(c)

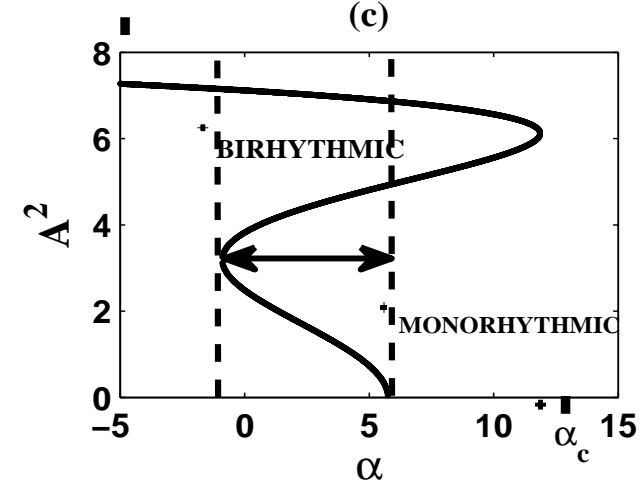

(b)

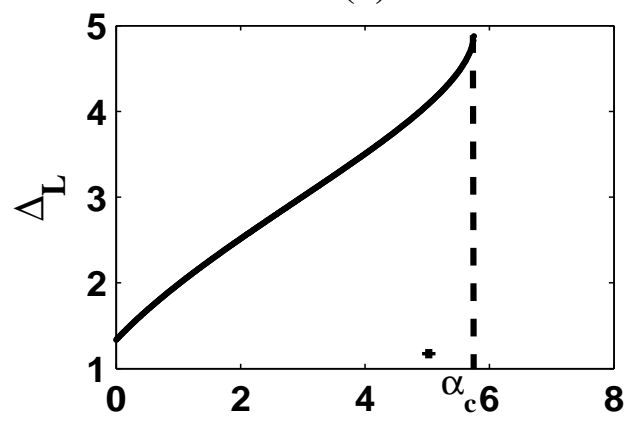

(d)

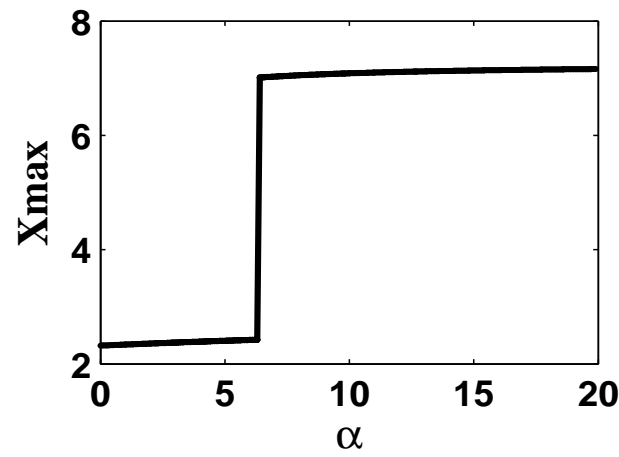

Figure 6: Map of $r$ versus $\alpha(a)$, length of birhythmic zone versus $\alpha(b)$, amplitude versus $\alpha(c)$, numerical bifurcation of maximas versus $\alpha(d)$. The parameters used are: $\gamma_{1}=35.0, r=0.0001, I=2.25$ and $S_{2}$

where $\omega_{n}(n=0,1,2, \ldots)$ are unknown constants at this step. By substituting eq. (6) and (7) in eq.(5) and finding the secular terms in different order of $\mu$, a solution of equation (5) can take this following approximation:

$$
x(t)=A \cos \omega t+\mu\left(\Gamma_{2} \sin \omega t+\Gamma_{3} \sin 3 \omega t+\Gamma_{4} \sin 5 \omega t+\Gamma_{5} \sin 7 \omega t\right)+O\left(\mu^{2}\right) .
$$

with

$$
\begin{aligned}
& \Gamma_{2}=\left(\frac{219}{3072} \alpha_{2} A^{7}-\frac{\alpha_{1}}{12} A^{5}+\frac{3}{32} A^{3}\right) ; \quad \Gamma_{3}=-\frac{1}{32}\left(\frac{9}{16} \alpha_{2} A^{7}-\frac{3}{4} \alpha_{1} A^{5}+A^{3}\right) \\
& \Gamma_{4}=-\frac{1}{384}\left(\frac{5}{4} \alpha_{2} A^{7}-\alpha_{1} A^{5}\right) ; \quad \Gamma_{5}=-\frac{1}{3072} \alpha_{2} A^{7} ;
\end{aligned}
$$

where the amplitude $A$ satisfies the following equation:

$$
1-\frac{1}{4} A^{2}+\frac{1}{8} \alpha_{1} A^{4}-\frac{5}{64} \alpha_{2} A^{6}=0,
$$

and the frequency $\omega$ is given by

$$
\omega=1+\mu^{2} \omega_{2}+O\left(\mu^{3}\right)
$$


with

$$
\begin{aligned}
\omega_{2}= & \frac{93}{65536} \alpha_{2}^{2} A^{12}-\frac{69}{16384} \alpha_{1} \alpha_{2} A^{10}+\left(\frac{67}{8192} \alpha_{2}+\frac{3}{1024} \alpha_{1}^{2}\right) A^{8}+\left(\frac{7}{2048} \theta_{2}-\frac{1}{96} \alpha_{1}\right) A^{6} \\
& +\left(-\frac{1}{48} \alpha_{1}+\frac{1}{128}\right) A^{4}+\frac{5}{64} A^{2} .
\end{aligned}
$$

\begin{tabular}{|l|c|c|c|l|}
\hline$S_{i}=\left(\theta_{1}, \theta_{2}\right)$ & Analytical Amplitude & Numerical Amplitude & Analytical Frequency & Numerical Frequency \\
\hline \multirow{3}{*}{$S_{1}=(0.03 ; 0.0007)$} & $A_{1}=2.860$ & $A_{1}=2.845$ & $\omega_{1}=1.000479$ & $\omega_{1}=0.9957$ \\
& $A_{2}=3.248$ & Unstable & $\omega_{2}=1.000487$ & Unstable \\
& $A_{3}=5.499$ & $A_{3}=5.494$ & $\omega_{3}=1.0003193$ & $\omega_{3}=0.9842$ \\
\hline \multirow{3}{*}{$S_{2}=(0.025 ; 0.0004)$} & $A_{1}=2.486$ & $A_{1}=2.476$ & $\omega_{1}=1.000465$ & $\omega_{1}=0.9880$ \\
& $A_{2}=3.821$ & Unstable & $\omega_{2}=1.000479$ & Unstable \\
& $A_{3}=7.116$ & $A_{3}=7.108$ & $\omega_{3}=0.997844$ & $\omega_{3}=0.9742$ \\
\hline \multirow{3}{*}{$S_{3}=(0.02 ; 0.0003)$} & $A_{1}=2.304$ & $A_{1}=2.301$ & $\omega_{1}=1.000455$ & $\omega_{1}=0.9888$ \\
& $A_{2}=5.001$ & Unstable & $\omega_{2}=1.000414$ & Unstable \\
& $A_{3}=6.772$ & $A_{3}=6.764$ & $\omega_{3}=1.000229$ & $\omega_{3}=0.9728$ \\
\hline \multirow{3}{*}{$S_{4}=(0.025 ; 0.0005)$} & $A_{1}=2.457$ & $A_{1}=2.446$ & $\omega_{1}=1.000463$ & $\omega_{1}=0.9881$ \\
& $A_{2}=4.322$ & Unstable & $\omega_{2}=1.000475$ & Unstable \\
\hline \multirow{3}{*}{$S_{5}=(0.02 ; 0.00025)$} & $A_{3}=5.694$ & $A_{3}=5.689$ & $\omega_{3}=1.000435$ & $\omega_{3}=0.9742$ \\
& $A_{1}=2.311$ & $A_{1}=2.308$ & $\omega_{1}=1.000456$ & $\omega_{1}=0.9888$ \\
& $A_{2}=4.586$ & Unstable & $\omega_{2}=1.000418$ & Unstable \\
& $A_{3}=8.065$ & $A_{3}=8.054$ & $\omega_{3}=0.995941$ & $\omega_{3}=0.9728$ \\
\hline
\end{tabular}

Table I: Comparison between analytical and numerical characteristics of the limit cycles. All data refer to the case $\gamma_{1}=100.0$.

The amplitude equation (9) can be solve using an algorithm of Newton-Raphson [24]. The results appearing in Figure 1 in the $\left(\theta_{1}, \theta_{2}\right)$ plane describe the area of one (unhatched) and three amplitudes (hatched) respectively. As shown experimental by Lechner and al. [6], the neuronal system (4) can emerged in two different stable attractors separate one to the other by an intermediate unstable attractor. This phenomena called bistability has been mentioned by many authors [7, 28]. The particular attractor (electrical mode) exhibited by the neuron is determined by initial conditions. Therefore the initial conditions of the model represent membrane potential and the states of the ion conductances [28]. Thus the attractor with small amplitude and high frequency characterize a subthreshold potential while the attractor with large amplitude and small frequency characterize an action potential as showed in [29].

A verification of the convergence of results has been evaluated in table I where the comparison between analytical and numerical results has been done, as well as the variation of the amplitude versus of $\theta_{1}$ and $\theta_{2}$ (such as in figure 2) for $\gamma_{1}=100.0$. The neuronal activity has been given in rest, spiking or bursting [16, 30, 31] and in different forms of coexistence states [7]. In our system, the flexibility of cell nervous is shows by the coexistence of two different oscillatory modes in amplitude and frequency. Figure 3 shows the coexistence between two regimes of self-sustained oscillations, as numerically shown by Lechner and al [6]. Bistability characterizing a existence to bifurcation by a coexistence of different modes (such as here electrical activities), give to neurons a rich forms of his information processing to generating pulse. We have in figure 4 the representation of the unstable attractor in dotted line and hatched part obtain using the floquet theory [7]. 
(a)

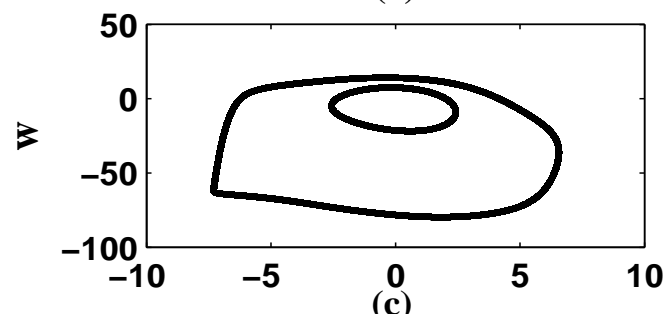

(c)

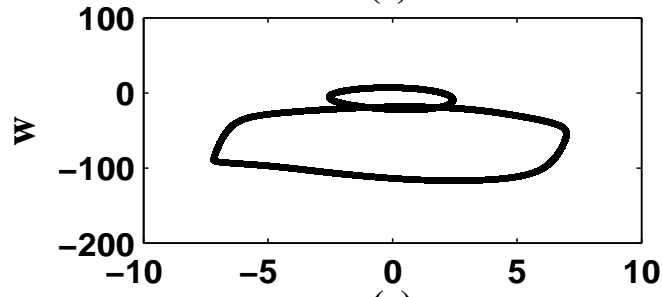

(e)

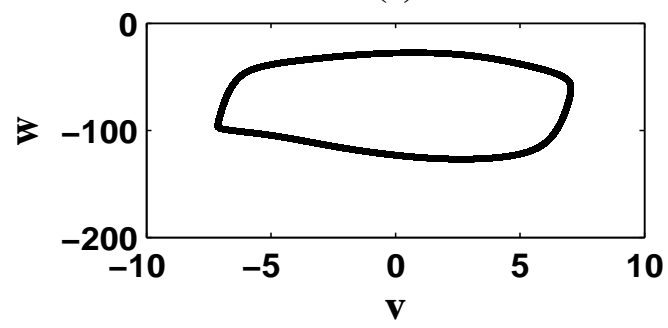

(b)
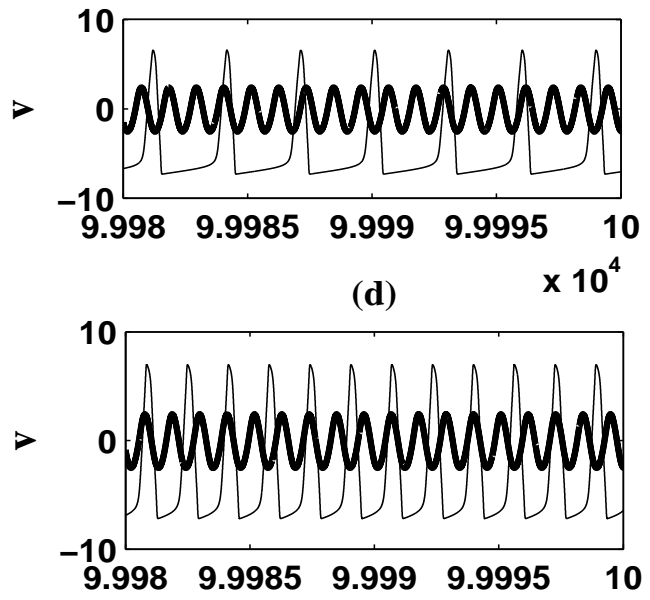

(f) $\times 10^{4}$

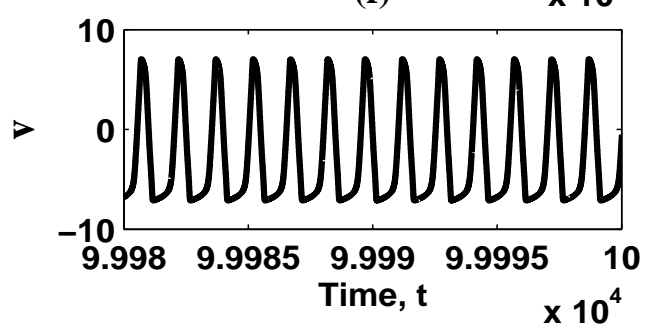

Figure 7: The coexistence between two regimes of self-sustained oscillations corresponding to the smaller (solid line, $A_{1}$, and frequency $\omega_{1}$ ) and larger (dotted line, $A_{3}$, and frequency $\omega_{3}$ ) limit cycles $(a),(b)$ for $\alpha=3.0 ;(c),(d)$ for $\alpha=5.0$; and existence of only one limit cycle $(e),(f)$ for $\alpha=7.0$ The parameters used are: $\gamma_{1}=35.0, r=0.0001, I=2.25, s=4.0$ and $S_{2}$.

\section{CONTROL OF BIRHYTHMICITY IN A CONDUCTANCE-BASED NEURONAL MODEL}

\section{A. Statement of the problem}

The problem of bidimensional neuron's models is that it does not give a real description to emission of membrane potential about the long time scale between action potential. It's showed that bursting cannot happen in two-variable [24], then the slow modulation of spiking during a burst (limitation to the time of emission to nerve impulse by a rest state or subthreshold oscillations) requires additional physical mechanism and dynamic variables. It's the reason for what HR[16] following Izhikevich[23] have added a third variable to stopped spiking modes. That is why in the main to have a real system, we add a slow processes ( channel affected by slow ion accumulation) to our idealized two-variables model and we obtain a three-dimensional birhythmic conductance-based neuronal model eq.(4). 


\section{B. Effects of adaptation variable with a constant external current}

Let us recall that $(\alpha \neq 0)$, the evolution of the dynamic of our neuronal system is now represented by the following three nonlinear differential system:

$$
\left\{\begin{array}{l}
\frac{d v}{d t}=2 v-\frac{1}{3} v^{3}+\theta_{1} v^{5}-\theta_{2} v^{7}+w-\alpha z+I, \\
\frac{d w}{d t}=1-\gamma_{1} v-5 v^{2}-w, \\
\frac{d z}{d t}=r(s v-z),
\end{array}\right.
$$

where $z(t)$ is an adaptation variable characterizing the slow flux ions current in the membrane. This parameter can be view as a low pass filter. $\alpha$ is the self-feedback strength and $r$ the cut-off frequency. Others parameters have the same signification as upper. It's clair that if $(\alpha=0)$ the slow $z$-variable becomes a parameter independent to the fast subsystem $v(t)$ and $w(t)$, we therefore have a two dimensional system without a third variable. To consider a system (4) and taking same considerations as the study of the precedent two dimensional equation, we obtain the following equation:

$\ddot{x}-\mu_{e f f}\left(1-x^{2}+\alpha_{1} x^{4}-\alpha_{2} x^{6}\right) \dot{x}+x=\mu_{e f f}^{2}\left(1-5 x^{2}-\frac{1}{3} x^{3}+\frac{1}{5} \alpha_{1} x^{5}-\frac{1}{7} \alpha_{2} x^{7}-\alpha(1-r) z\right)+I_{e x t}$,

where,

$$
\begin{aligned}
\mu_{e f f} & =\frac{1}{\omega_{e f f}}, \quad \alpha_{1}=5 \theta_{1}, \quad \alpha_{2}=7 \theta_{2}, \quad \omega_{e f f}=\sqrt{\gamma_{1}-2+\alpha r s}, \\
v(t) & =x, \quad I(t)=\frac{1}{\omega_{e f f}^{2}} I\left(\frac{t}{\omega_{e f f}}\right)=I_{e x t} .
\end{aligned}
$$

An appropriate analytical tool to understand the effect of $r$ and $\alpha$ on the dynamic of the system, is the harmonic decomposition method [25]. Let us assume the approximate solution of [13] as:

$$
x(t)=A \cos \left(\omega t+\phi_{1}\right)
$$

and

$$
z(t)=B \cos \left(\omega t+\phi_{2}\right)
$$

where, $\mathrm{A}, \omega, \phi_{1}$ respectively the natural amplitude, frequency and phase of the oscillator. $\mathrm{B}, \omega, \phi_{2}$ respectively the amplitude, frequency and phase of the filter. Therefore, considering a phase difference $\phi=\phi_{2}-\phi_{1}$ and letting $\phi_{1}=0$ for convenience, we can written solution of eq. 13 as:

$$
x(t)=A \cos \omega t
$$

and

$$
z(t)=B \cos (\omega t+\phi)
$$


(a)

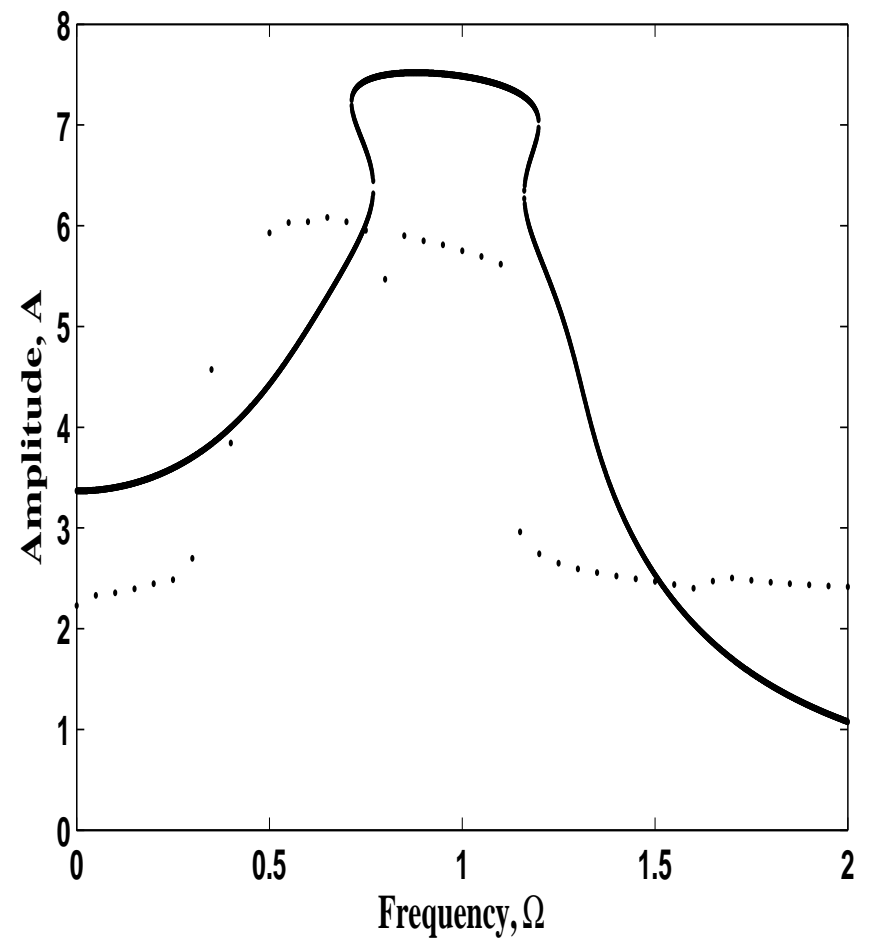

Figure 8: Comparison between analytical (with lines) and numerical(with dots) frequency-response curves , for $\alpha=0.0$ The parameters used are: $\gamma_{1}=35.0, E=2.25, s=4.0$ and $S_{2}$

Substitution of (16) and (17) into (13), not taking into account the higher harmonics terms considered as forcing terms [32], we obtain:

$$
\begin{aligned}
& \left(1-\omega^{2}+\mu_{e f f} \frac{\alpha r^{2} s(1-r)}{r^{2}+\omega^{2}}+\mu_{\text {eff }}^{2}\left(\frac{1}{8} A^{2}-\frac{\alpha_{1}}{8} A^{4}+\frac{5}{64} \alpha_{2} A^{6}\right)\right) A \cos \omega t= \\
& \mu_{e f f} \omega\left(\left(1-\frac{1}{4} A^{2}+\frac{\alpha_{1}}{8} A^{4}-\frac{5}{64} \alpha_{2} A^{6}\right)-\mu_{e f f} \frac{s r \alpha(1-r)}{r^{2}+\omega^{2}}\right) A \sin \omega t \\
& +H \text { (higher harmonicterms) }
\end{aligned}
$$

and

$$
\frac{B}{A}=\frac{s r}{\sqrt{r^{2}+\omega^{2}}}
$$

Let us assume as [32] that the amplitude $B$ of the filtered signal, $z(t)$, remains almost the same as the original of the signal $x(t)$, but different phase $\phi$. Using this last consideration, (19) into (18) lead us respectively to the following frequency and amplitude equation

$$
1-\omega^{2}+\mu_{e f f} \frac{\alpha r(1-r)}{\sqrt{r^{2}+\omega^{2}}}+\mu_{e f f}^{2}\left(\frac{1}{8} A^{2}-\frac{\theta_{1}}{8} A^{4}+\frac{5}{64} \theta_{2} A^{6}\right)=0,
$$

and

$$
1-\frac{1}{4} A^{2}+\frac{\alpha_{1}}{8} A^{4}-\frac{5}{64} \alpha_{2} A^{6}-\mu_{e f f} \frac{\alpha(1-r)}{\sqrt{r^{2}+\omega^{2}}}=0,
$$


(a)

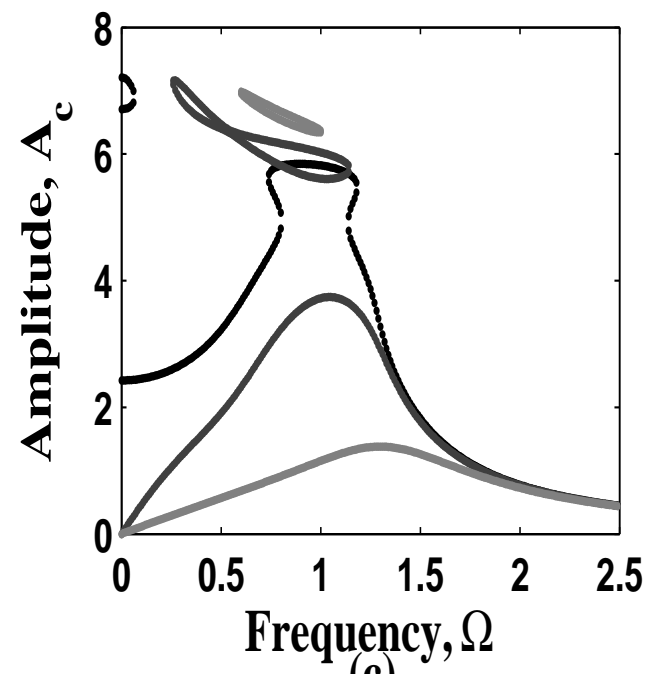

(c)

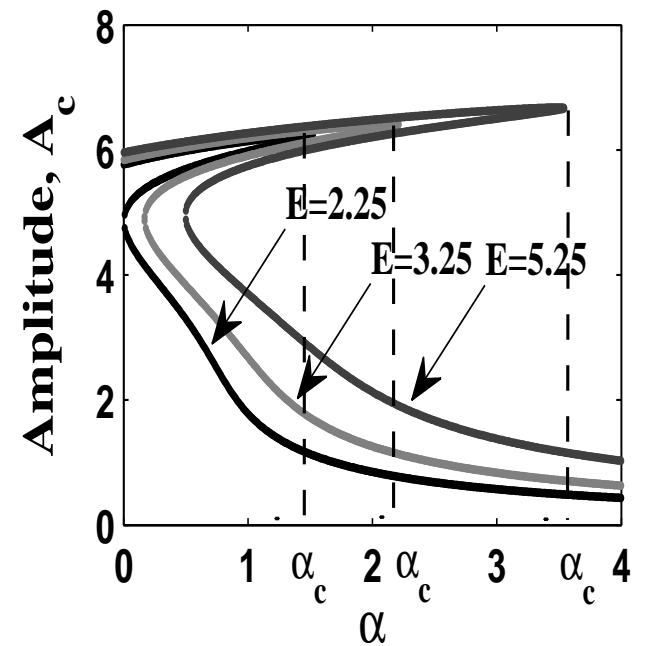

(b)

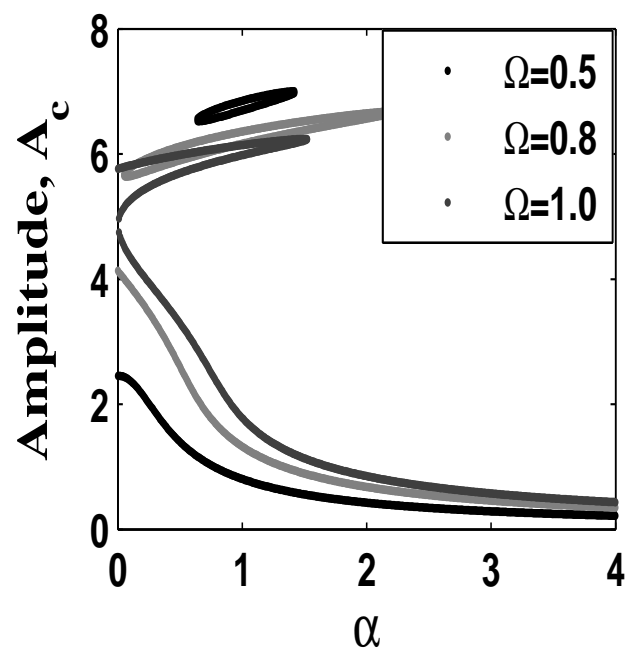

(d)

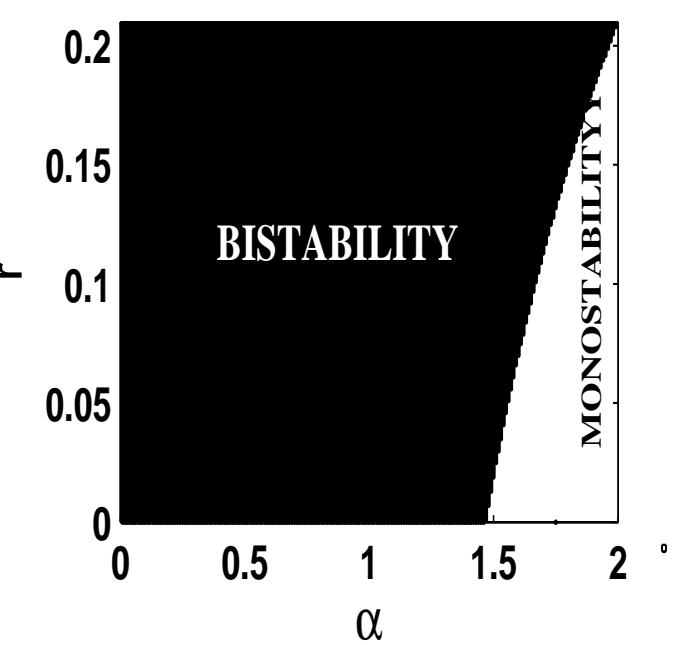

Figure 9: Response curves versus $\Omega$ for different values of $\alpha(a)$ and versus $\alpha$ for different values of $\Omega(b)$ and $E(c)$. Map of $r$ versus $\alpha(d)$. The parameters used are: $\gamma_{1}=35.0, E=2.25, s=4.0, r=0.0001$, $\Omega=1$ and $S_{2}$

we observe that for $\alpha=0$ or $r=1, \omega^{2} \simeq 1$ and eq. 21 is the same to the amplitude equation 96 obtain in the birhythmic oscillator (5) in the absence of feedback. For other case, equation (20) and equation (21) depends to interaction between $\alpha$ and $\mathrm{r}$; therefore the system have an effective natural frequency and amplitude. The frequency in the harmonic limit reduces to $\omega=1$, the three limit cycles (two stable, one unstable) are results of the three roots of the amplitude equation. To get an idea of the amplitude of the limit cycles, we analyze the stability of the system using the energy balance method [32]. In order to analyze our 
(a)
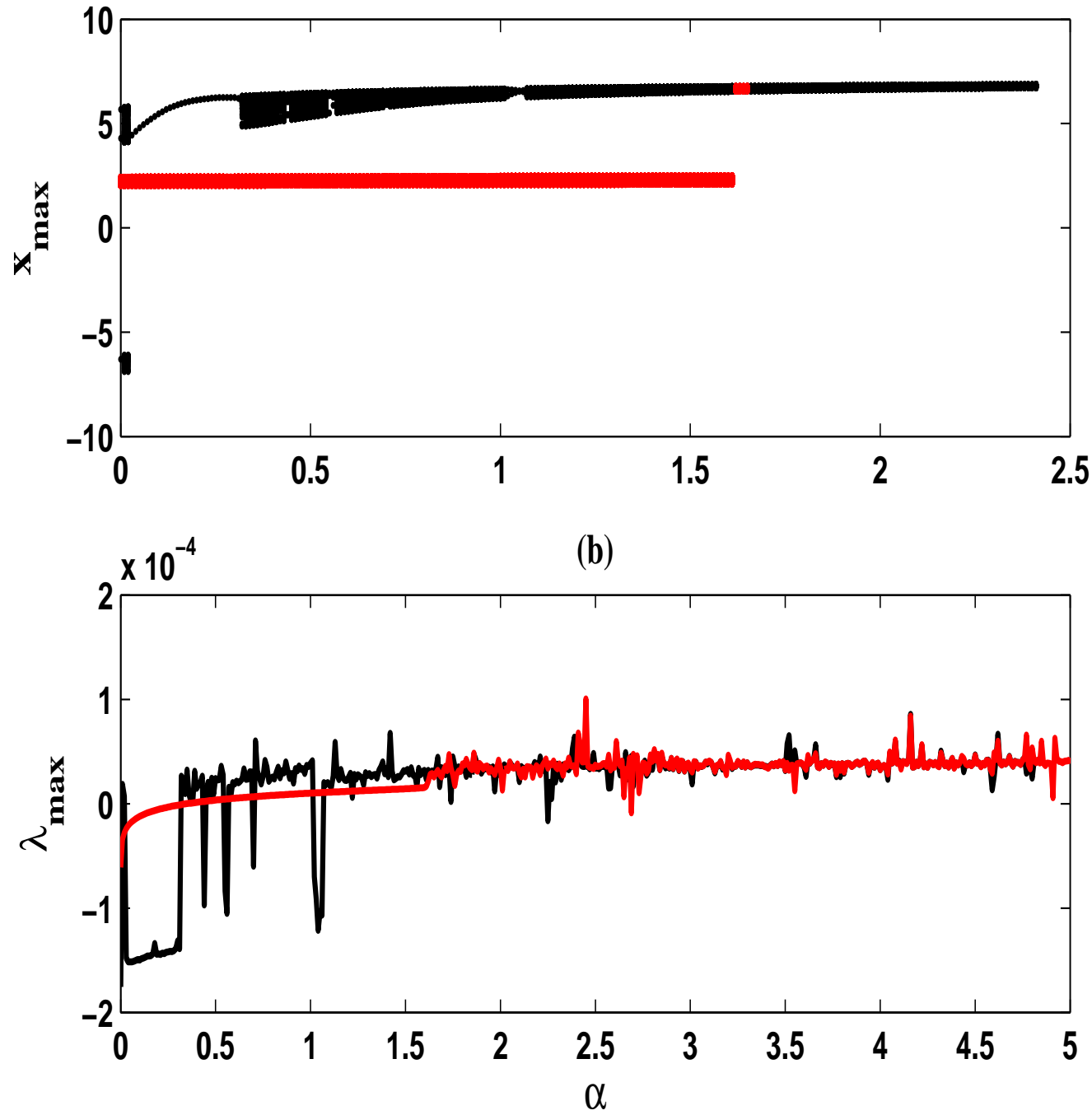

Figure 10: Bifurcation and lyapunov diagram respectively(a) and (b) versus a control parameter $\alpha$ for $r=0.0001$ and The parameters used are: $\gamma_{1}=35.0, E=2.25, s=4.0$ and $S_{2}:(a),(b)$

system (5), we rewrite equation (13) as the following equation:

$$
\ddot{x}-\mu_{e f f} h(x) \dot{x}+x+\mu_{e f f}^{2} \alpha(1-r)\left(s x-\frac{\dot{z}}{r}\right)=\mu_{e f f}^{2}\left(1-5 x^{2}-\frac{1}{3} x^{3}+\frac{1}{5} \alpha_{1} x^{5}-\frac{1}{7} \alpha_{2} x^{7}+I_{e x t}\right),
$$

The solution of equation 22 for $\mu_{e f f}=0$ is given by:

$$
x(t)=A \cos t
$$

and

$$
z(t)=A \cos (t+\phi)
$$

The change energy $\Delta E$ in one period $0 \leq t \leq T$ is found by considering the term $[25]$ as the external forcing term. 
(a)

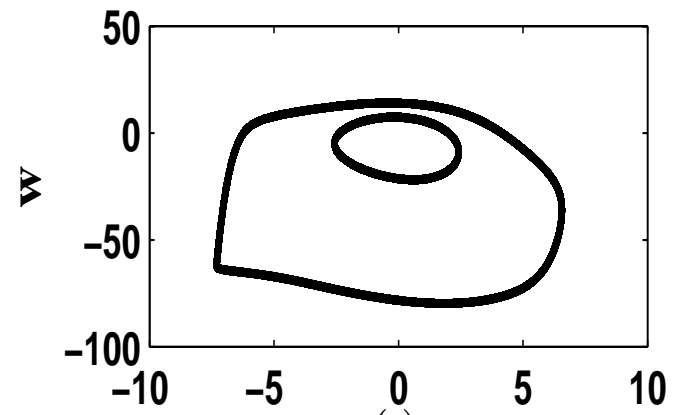

(c)

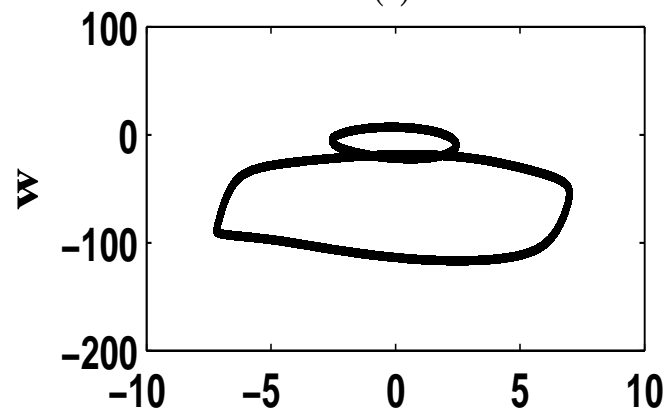

(e)

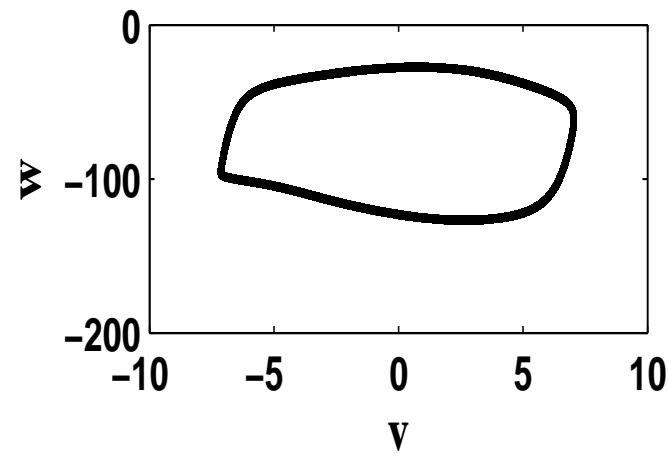

(b)
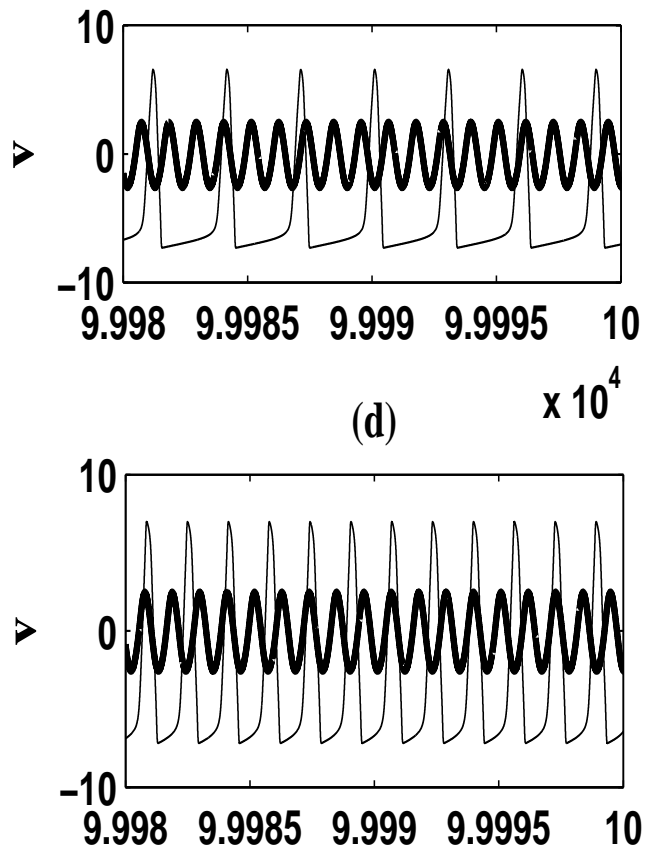

(f)

$\times 10^{4}$

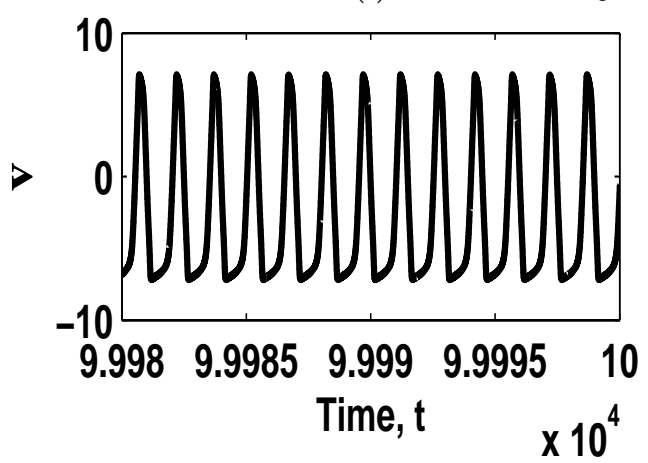

Figure 11: Map of a coexistence to period-11 subthreshold oscillation and spiking mode $\alpha=0.28(a)$, coexistence of chaotic subthreshold oscillation and period-2 bursting $\alpha=1.03(b)$, coexistence to chaotic subthreshold oscillation and period-3 bursting $\alpha=0.56(c)$, coexistence to chaotic subthreshold oscillation and period-4 bursting $\alpha=0.44(d)$, coexistence to chaotic subthreshold oscillation and chaotic bursting $\alpha=0.46(e)$, existence to a monostable chaotic bursting $\alpha=1.71(e)$; for the two initial conditions $(2,2,5)$ and $(5,5,5)$ The parameters used are: $\gamma_{1}=35.0, E=2.25, s=4.0, r=0.0001$ and $S_{2}$

$$
\mu_{e f f} h(x) \dot{x}+\mu_{e f f}^{2}\left(1-5 x^{2}-\frac{1}{3} x^{3}+\frac{1}{5} \alpha_{1} x^{5}-\frac{1}{7} \alpha_{2} x^{7}-\alpha(1-r)\left(s x-\frac{\dot{z}}{r}\right)+I_{e x t}\right)
$$


(a)

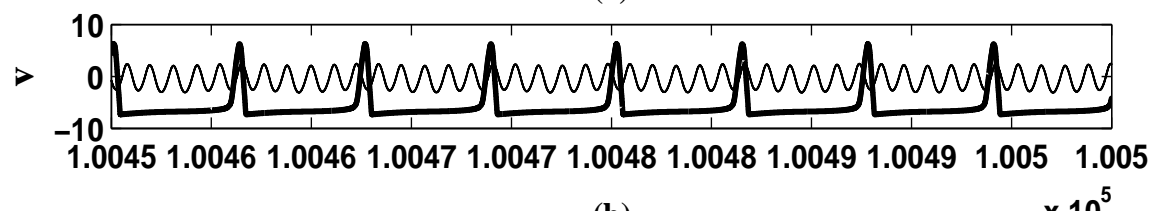

(b)

$\times 10^{5}$

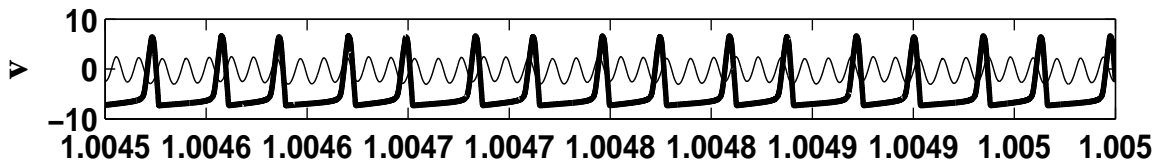

(c)

$\times 10^{5}$

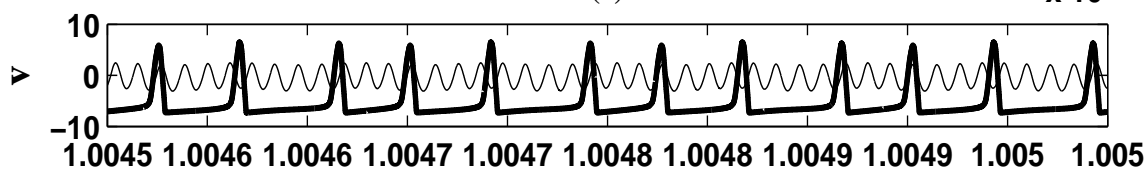

(d)

$\times 10^{5}$

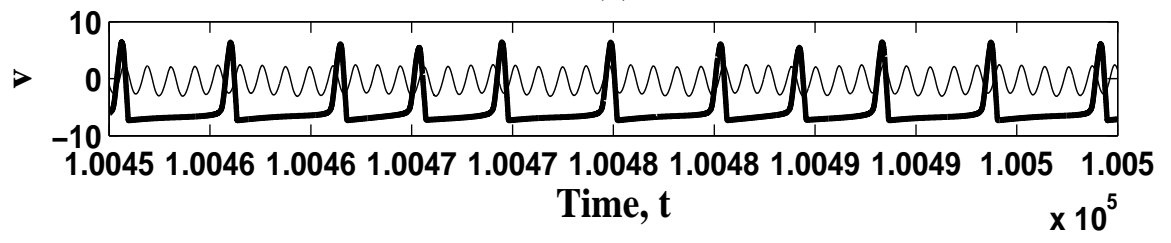

(e)

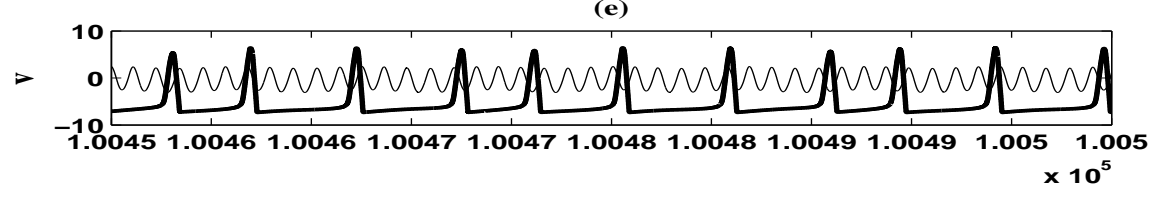

(f)

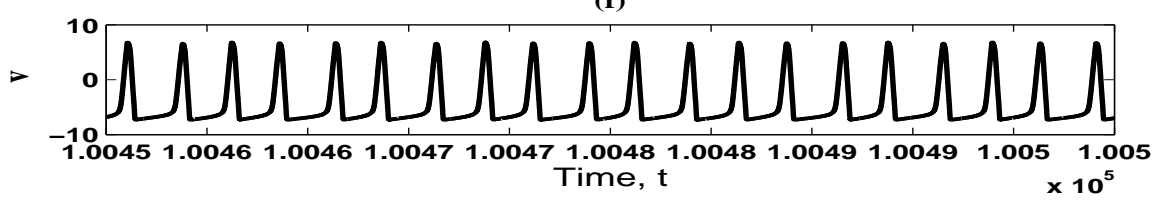

Figure 12: Times series of a coexistence to period-11 subthreshold oscillation and spiking mode $\alpha=$ $0.28(a)$, coexistence of chaotic subthreshold oscillation and period-2 bursting $\alpha=1.03(b)$, coexistence to chaotic subthreshold oscillation and period-3 bursting $\alpha=0.56(c)$, coexistence to chaotic subthreshold oscillation and period-4 bursting $\alpha=0.44(d)$, coexistence to chaotic subthreshold oscillation and chaotic bursting $\alpha=0.46(e)$, existence to a monostable chaotic bursting $\alpha=1.71(e)$; for the two initial conditions $(2,2,5)$ and $(5,5,5)$ The parameters used are: $\gamma_{1}=35.0, E=2.25, s=4.0, r=0.0001$ and $S_{2}$ 
(a)
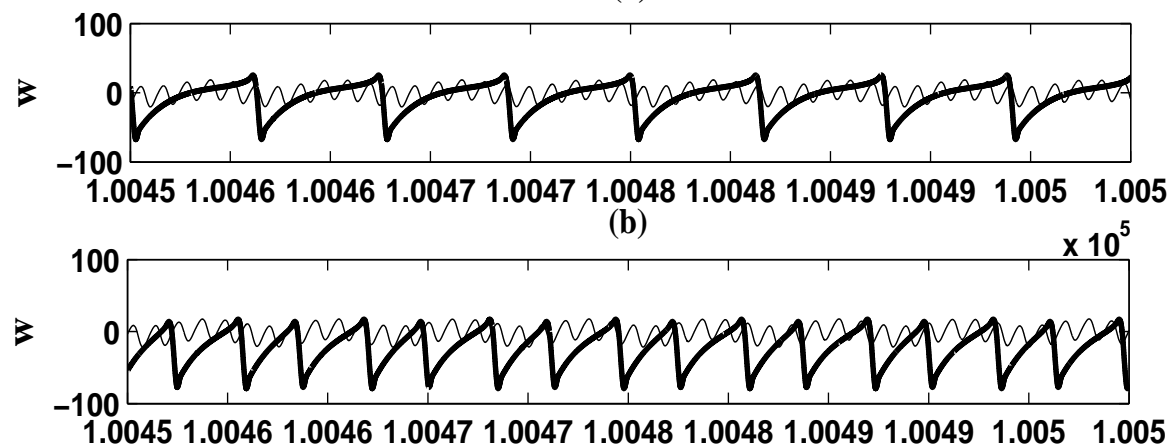

(c)

$\times 10^{5}$

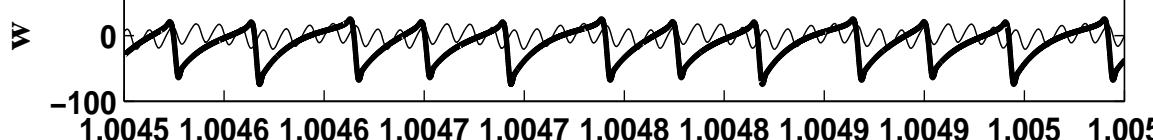

1.00451 .00461 .00461 .00471 .00471 .00481 .00481 .00491 .00491 .0051 .005

100

(d)

$\times 10^{5}$

3

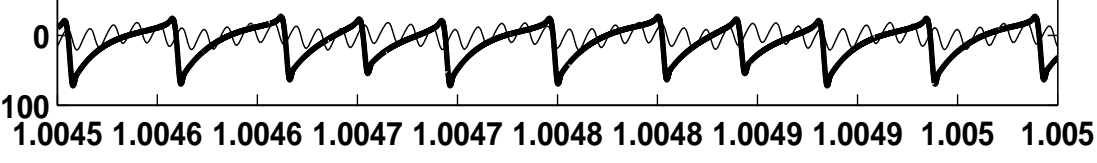

$\times 10^{5}$

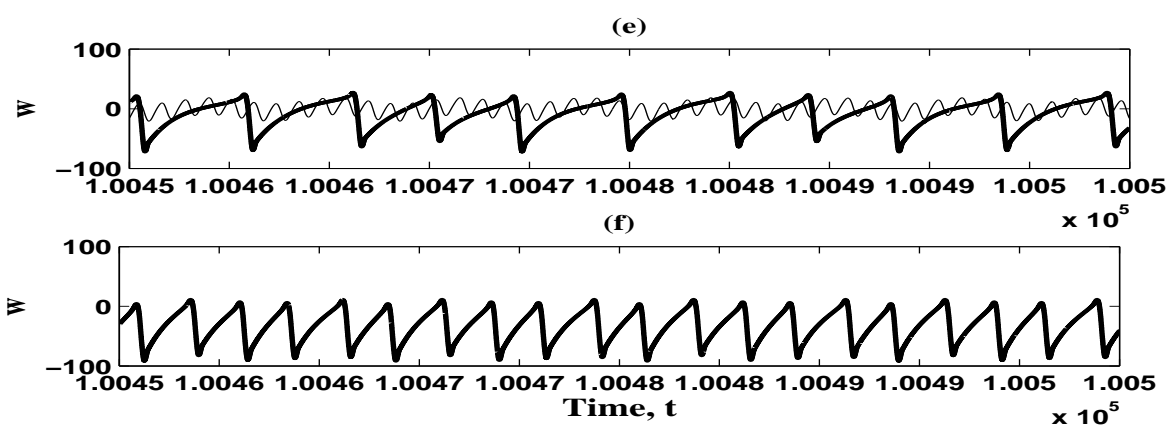

Figure 13: Times series of a coexistence to period-11 subthreshold oscillation and spiking mode $\alpha=$ $0.28(a)$, coexistence of chaotic subthreshold oscillation and period-2 bursting $\alpha=1.03(b)$, coexistence to chaotic subthreshold oscillation and period-3 bursting $\alpha=0.56(\mathrm{c})$, coexistence to chaotic subthreshold oscillation and period-4 bursting $\alpha=0.44(d)$, coexistence to chaotic subthreshold oscillation and chaotic bursting $\alpha=0.46(e)$, existence to a monostable chaotic bursting $\alpha=1.71(e)$; for the two initial conditions $(2,2,5)$ and $(5,5,5)$ The parameters used are: $\gamma_{1}=35.0, E=2.25, s=4.0, r=0.0001$ and $S_{2}$ 
Therefore the change energy is given by:

$$
\begin{aligned}
\Delta E & =E(T)-E(0) \\
& =\int_{0}^{T} \dot{x} d t\left(\mu_{e f f} h(x)+\mu_{e f f}^{2}\left(1-5 x^{2}-\frac{1}{3} x^{3}+\frac{1}{5} \alpha_{1} x^{5}-\frac{1}{7} \alpha_{2} x^{7}-\alpha(1-r)\left(s x-\frac{\dot{z}}{r}\right)\right)\right)
\end{aligned}
$$

Thus, equations (23) and (24) into equation (26) gives:

$$
h\left(A^{2}\right)=1-\frac{1}{4} A^{2}+\frac{\alpha_{1}}{8} A^{4}-\frac{5}{64} \alpha_{2} A^{6}-\mu_{e f f} \frac{\alpha(1-r)}{\sqrt{r^{2}+1}}=0
$$

The presence of limit cycle occur for $\Delta E=0$, that's why the presence of limit cycles and their stability can be determined by solving equation 27). Then to do this, we plot $h\left(A^{2}\right)$ versus $A$. A stability of the limit cycle is determined by the slope of the curve $h\left(A^{2}\right)$ versus $A$ at the zero crossing points. The negative slope determin the stable limit cycle, thus we can write

$$
\left.\frac{d \Delta E(A)}{d A}\right|_{\text {limit cycle }}<0
$$

as the condition of a stable limit cycle. It's so evident as showed by the floquet theory that for a birhythmic dynamic, the second amplitude is unstable. Figure 5 shows the curves of $h\left(A^{2}\right)$ versus amplitude $A$ for different values of $\alpha$, with $r=0.0001$ (see Fig 5 (a)) and for different values of $\mathrm{r}$, with $\alpha=7.0$ (see Fig $5(\mathrm{~b}))$. One finds that, for fixed range of $r$, the graph is displaced upward with the increases of $r$. However, an opposite effect is observed when increasing the coupling parameter $\alpha$. It is important to note that the slow variable called here feedback parameter, plays an important role on the existence and stability of the three amplitude of our new model. The map in figure 6(a) resume the interaction with $r$ and $\alpha$ and show the couple ( $\alpha, \mathrm{r})$ in which we can have one solution (monorhythmic mode) or three solutions (birhythmic mode). The hatched and unhatched regions in figure 6 a) show respectively the area of existence of three solutions (not effect of filtered-feedback then permeability to potassium $\mathrm{K}^{+}$or sodium $\mathrm{Na}^{+}$ions) and one solution (effect of filtered then strong permeability only to sodium $\mathrm{Na}^{+}$ions). It's also shown in figure 6(a),(b),(c) that when $\alpha>\alpha_{c}$, it's possible to have effect of filtered-feedback. But when $r>r_{c}$, there is no effect of filtered-feedback for all values of $\alpha$. Therefore, effect of $z(t)$ on the dynamic of the system strongly depend on value of $r$. Large values of $r$ have no filtering effect, that is the neuron can stay to one subthreshold oscillation or to emission of action potential depending a state of ion conductance. For small values of $r$, there's strong possibility filtering effect in relation to it self-strength feedback $\alpha$. That is why H.R[16], Rinzel and Ermentrout[24], Andrey and Cymbalyak[28] said in their dynamic's studies that only $\mathrm{r}$ taken in range $0<r \ll 1$ can permit to make $z(t)$ a slow variable than the fast variable $v$ and $w$ to have an effect on the dynamic.

In order to verified our analytical results, we firstly show in figure 6(d) the bifurcation diagram obtained numerically which confirm the coexistence of only regular states and the switching at the critical value of $\alpha$. Secondly, we show in figure7, the evolution of membrane potential for two couple of $(\alpha, \mathrm{r})$ taken in map of the figure 6 with initial condition $\left(v_{0}, w_{0}, z_{0}\right)$. When $(\alpha, r)=(1.0,0.0001)$ and with the two following initial 
(a)

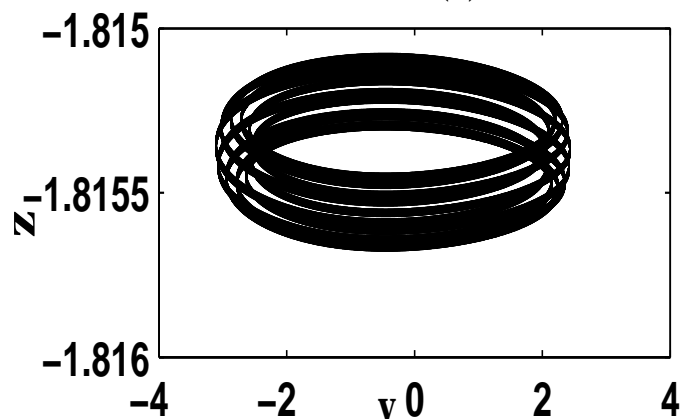

(c)

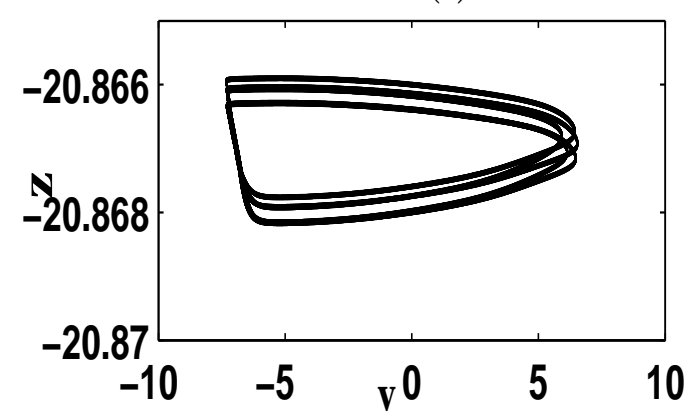

(e)

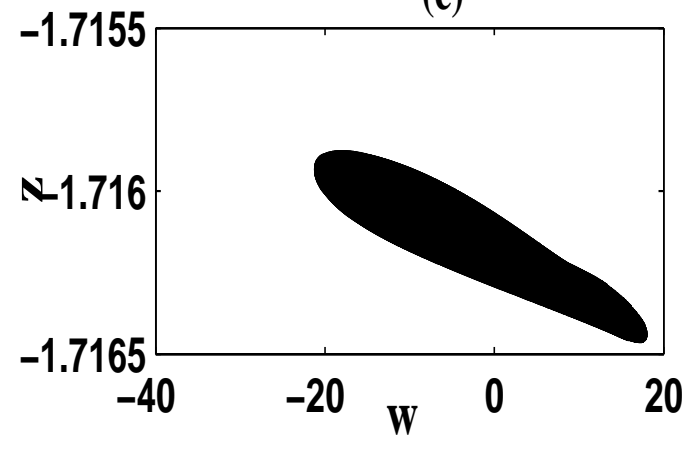

(b)

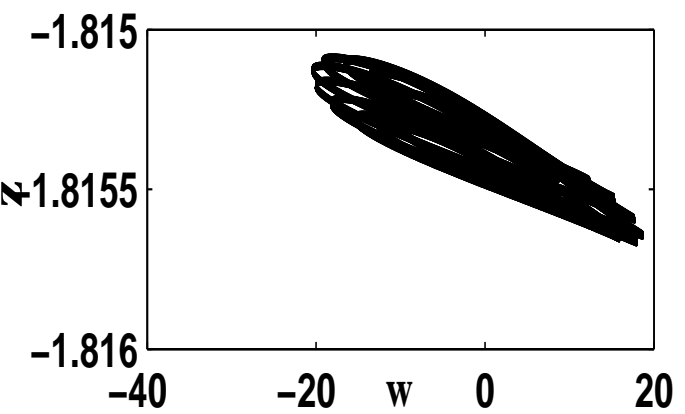

(d)

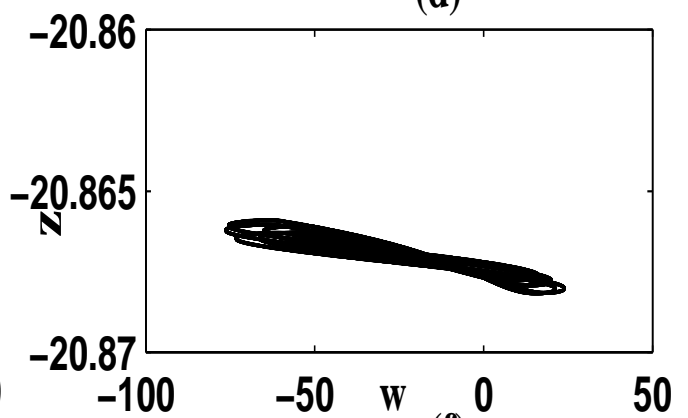

(f)

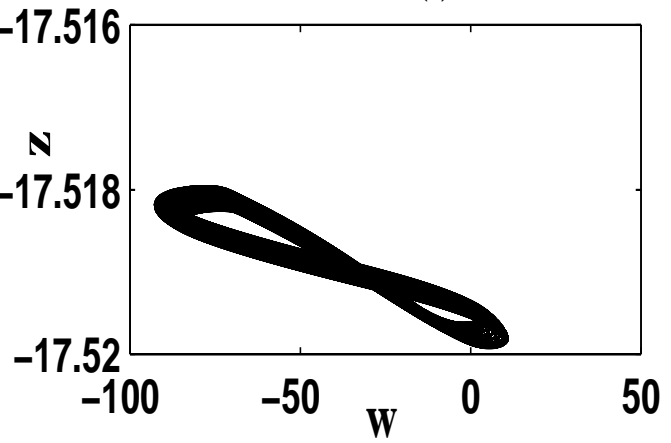

Figure 14: Phase portrait profils for $\alpha=0.28(a),(b)$ and $\alpha=0.7(e)$ with $(2,2,5)$ as initial condition; $\alpha=0.7(c),(d)$ and $\alpha=0.7(f)$ with $(5,5,5)$ as initial condition. The parameters used are: $\gamma_{1}=35.0$, $E=2.25, s=4.0, r=0.0001$ and $S_{2}$

conditions: $\left(v_{0}, w_{0}, z_{0}\right)=(2.0,2.0,5.0)$; and $\left(v_{0}, w_{0}, z_{0}\right)=(5.0,5.0,5.0)$, we obtain the same dynamic oscillations as in figure 3 consequence, no effect of filtered-feedback. When $(\alpha, r)=(7.0,0.0001)$, the oscillation with small amplitude $A_{1}$ called (subthreshold potential) is suppressed and the dynamic of system is only in the attractor of large amplitude $A_{3}$ (called nerve impulse). This only in spiking mode for any initial condition as shown by Andrey and Cymbalyak[28] and in a Jerk systems[17]. 


\section{Simultaneous effect of filtered-feedback and external variable current}

In the goal to approach the near of possible a three dimensional autonomous Moris-Lecar Model[23] about characteristics as many complex forms of coexisting and control of multistable states, our system equation (12) need an additional modulation. We consider here that the intrinsic nonlinear terms avoided in our simple model (3) will be taken into account here as a forcing terms which modulate the constant external current applied in a sinusoidal form. Let us then consider the following system:

$$
\begin{aligned}
& \left\{\begin{array}{l}
\ddot{x}-\mu_{1}\left(1-x^{2}+\alpha_{1} x^{4}-\alpha_{2} x^{6}\right) \dot{x}+x=\mu_{1}^{2}\left(1-5 x^{2}-\frac{1}{3} x^{3}+\frac{1}{5} \alpha_{1} x^{5}-\frac{1}{7} \alpha_{2} x^{7}-\alpha(1-r) z\right)+I_{e x t} \\
\dot{z}=r(s x-z)
\end{array}\right. \\
& \mu_{1}=\frac{1}{\omega_{1 e f f}}, \quad \alpha_{1}=5 \theta_{1}, \quad \alpha_{2}=7 \theta_{2}, \quad \omega_{1 e f f}=\sqrt{\gamma_{1}-2+\alpha r s}, \\
& v(t)=x, \quad I_{\text {ext }}=E \cos (\Omega t) .
\end{aligned}
$$

With a sinusoidal force, the amplitude of the forced harmonic oscillatory states can be found using the harmonic balance method [32]. Assuming that the fundamental component of the solutions has the period of the external excitation, we express the solution $x$ as:

$$
x(t)=a_{1} \cos \Omega t+a_{2} \sin \Omega t=A_{c} \cos (\Omega t-\phi)
$$

By substituting equation (29) into equation (28), we obtain a particular solution of a variable $z$ as:

$$
z(t)=\left(\Gamma_{1} a_{1}-\Gamma_{2} a_{2}\right) \cos \Omega t+\left(\Gamma_{1} a_{2}-\Gamma_{2} a_{1}\right) \sin \Omega t,
$$

where,

$$
\Gamma_{1}=\frac{r}{r^{2}+\Omega^{2}}, \quad \Gamma_{2}=\frac{\Omega}{r^{2}+\Omega^{2}} .
$$

By substuting equation (30) into the system (28), it becomes:

$$
\ddot{x}-\mu_{2}\left(1+d-x^{2}+\alpha_{1} x^{4}-\alpha_{2} x^{6}\right) \dot{x}+x=\mu_{2}^{2}\left(1-5 x^{2}-\frac{1}{3} x^{3}+\frac{1}{5} \alpha_{1} x^{5}-\frac{1}{7} \alpha_{2} x^{7}\right)+E \cos (\Omega t),
$$

where,

$$
\mu_{2}=\frac{1}{\omega_{2 e f f}}, \quad \omega_{2 e f f}=\sqrt{\gamma_{1}-2+\alpha\left(\Gamma_{1}(1-r)+r s\right)}, \quad d=\frac{\alpha(1-r)}{\mu_{2}\left(r^{2}+\Omega^{2}\right)} .
$$

Inserting equation (29) into equation (31) and equating the coefficient of the cosine and sine terms separately, the amplitude of the oscillatory states satisfies the following algebraic equation:

$$
\begin{aligned}
& \frac{25}{4096} \alpha_{2}^{2} A_{c}^{14}-\frac{5}{256} \alpha_{1} \alpha_{2} A_{c}^{12}+\left(\frac{5}{128} \alpha_{2}+\frac{1}{64} \alpha_{1}^{2}\right) A_{c}^{10}-\left(\frac{5}{32}\left(1+\frac{\alpha(1-r)}{\mu_{2}\left(r^{2}+\Omega^{2}\right)}\right) \alpha_{2}+\frac{1}{16} \alpha_{1}\right) A_{c}^{8} \\
& +\left(\frac{1}{4}\left(1+\frac{\alpha(1-r)}{\mu_{2}\left(r^{2}+\Omega^{2}\right)}\right) \alpha_{1}+\frac{1}{16}\right) A_{c}^{6}-\frac{1}{2}\left(1+\frac{\alpha(1-r)}{\mu_{2}\left(r^{2}+\Omega^{2}\right)}\right) A_{c}^{4} \\
& +\left(\left(1+\frac{\alpha(1-r)}{\mu_{2}\left(r^{2}+\Omega^{2}\right)}\right)^{2}+\frac{\left(1-\Omega^{2}+\mu_{2}^{2}\left(-\frac{1}{4} A_{c}^{2}+\frac{1}{8} \alpha_{1} A_{c}^{4}-\frac{5}{64} \alpha_{2} A_{c}^{6}\right)\right)^{2}}{\mu_{2}^{2} \Omega^{2}}\right) A_{c}^{2} \\
& -\frac{E^{2}}{\mu_{2}^{2} \Omega^{2}}=0,
\end{aligned}
$$


where,

$$
\begin{aligned}
A_{c}^{2} & =a_{1}^{2}+a_{2}^{2}, \\
\tan \phi & =\mu_{2} \Omega \frac{\left(1-\frac{1}{4} A_{c}^{2}+\frac{1}{8} \alpha_{1} A_{c}^{4}-\frac{5}{64} \alpha_{2} A_{c}^{6}+\frac{\alpha(1-r)}{\mu_{2}\left(r^{2}+\Omega^{2}\right)}\right)}{\left(\Omega^{2}-1+\mu_{2}^{2}\left(-\frac{1}{4} A_{c}^{2}+\frac{1}{8} \alpha_{1} A_{c}^{4}-\frac{5}{64} \alpha_{2} A_{c}^{6}\right)\right)} .
\end{aligned}
$$

In the absence of adaptation variable $(\alpha=0)$, we plot in figure 8 the analytical and numerical results of the frequency responses-curves versus the frequency $\Omega$. This curve at $\Omega=1$, reveal the resonant properties to the neuronal system due to the presence of subthreshold oscillations. It's appear that although a forced terms considered, the model can exhibit one or three limit cycle as the autonomous system. In the presence of an adaptation variable $(\alpha \neq 0)$, we also present the response curve of the system versus frequency $\Omega$ for different values of the self-feedback strength $\alpha$ figure 9 (a) when $E$ is fixed. It appear that the parameter $\alpha$ control the resonance frequency and amplitude's peak of the system. In fact, when $\alpha$ increase, the resonance's peak decrease until damped completely while it corresponding frequency increase. Figure 9 (b) gives the response curve versus the self-feedback strength $\alpha$ for some values of frequency of external stimulation $\Omega$. It appear there that, the system has a hysterical behavior when the external frequency is not taken in resonance $(\Omega=1)$. Figure 9 (c) gives the response curve versus $\alpha$ for some values of intensity of external stimulation $E$.

The phenomenon of hysteresis and the switching to the system by suppressing to one stable attractor when $\alpha$ reaches a critical value $\alpha_{c}$ (for example, $\alpha_{c} \sim 1.5$ for $\Omega=1$ and $E=2.25$ ), is observed. Figure 9(d) show in the map of $r$ versus $\alpha$, a coexistence of two different electrical modes ( bistability in hatched part ) or a existence of only one electrical mode ( monostability in non-hatched part ). In order to confirm the behavior obtain by the analytical study, a numerical study using a system (28) is analysed by the means of bifurcation and his corresponding lyapunov exponents diagrams represented in figures 10 (a),(b). These figures show clearly the coexistence of two different forms of attractors (periodic or chaotic) got respectively with the initial conditions $(2,2,5)$ (red) and $(5,5,5)$ (black). When $\alpha$ varies, the neuron present a coexistence with various complex and captivating behavior such as period, period-doubling cascades, and chaos which prove a variability to frequency. At the critical value of $\alpha_{c} \approx 1.6$ the system tune from the bistability to monostability for any initial condition, which proves the switching predict in analytical analysis.

These results were founded by Cymbalyuk and Shilnikov [28] and in the three dimensional autonomous ML model[12] but coexisting attractors were in the same amplitude. They were also founded in a simple and chaotic Jerk system[17], in the chua's system[19] and in a Yu-Wang four-Wing chaotic system[20]. By taking some value of the control parameter $\alpha$, we shows in figure 11 with some phase portrait, the dynamic of the neuron as: a coexistence to period-11 substhreshold oscillations and tonic spiking figure 11.a); a coexistence to tonic chaotic substhreshold oscillations and periodic bursting with two, three, four spike per burst figure 11 (b),(c),(d); a coexistence to tonic chaotic substhreshold oscillations and chaotic bursting 
figure 11(e). Figure 11(f) show a existence to only one chaotic attractor specially with large amplitude. This large amplitude is therefore desirable because it can become more chaotic. The corresponding times series are done in figures 12 and 13 . Figure 14 shows others profiles obtained in the others phase plane for some values of a control parameter $\alpha$ and initial conditions. Variable $z(t)$ characterising slow flux ions in membrane play an important role in neuron because it can be considered as a filtered. It permit with his bifurcation parameter $\alpha$ to neuron, to moved in attractor who can change significatively and where his efficience is not limited in his process of transmission of information. This control of the neuronal dynamic may throw new light on the diagnosis and treatment of neurological diseases.

\section{CONCLUSION}

In summary, we have proposed firstly in this paper, a three dimensional conductance-based neuronal model inspired by an improving of M-L system [12]. we have show that his autonomous dynamic without adaptation variable is the same as a modified van der pol system, and depend strongly to nonlinear coefficients $\theta_{1}$ and $\theta_{2}$ function of conductance parameter. By using the Lindsted's perturbation method [26], with an appropriate parameters $\theta_{1}$ and $\theta_{2}$, we have found a coexistence of two different attracting modes in frequency and amplitude (bistability) function to initial condition. This behavior characterize the neuron as a system with rich forms of information processing principally here in subthreshold oscillation (small amplitude $A_{1}$ ) or in emission of nerve impulse (large amplitude $A_{3}$ ) separated by an unstable attractor $A_{2}$ named threshold.

Secondly, to make a system realistic, we we inspired to the concept of slow-fast variable in coupled our first bidimensional mathematical model with an appropriate variable called adaptation variable. This with coupled parameter $\alpha$ due to ions accumulation in the membrane cellular. We have then quantified the effect of this third variable with a forcing term on our autonomous dynamic by the harmonic balance method [32]. We have shown that neuron admit a membrane potential resonance with a resonance frequency which can be controlled by a control parameter $\alpha$. At the resonance, the bifurcation and lyapunov exponent diagrams versus $\alpha$, have show a coexistence of two complex attractors namely periodic, period doubling and chaotic.

These are the sign to the presence of biological behavior as tonic spiking, periodic bursting, chaotic spiking and bursting respectively. For $\alpha \geq 1.6$, neuron judge necessary to switch his activity from a state of two coexisting attractors to monostability in also a attractor with large amplitude (action potential). This by a suppressing of the attractor with small amplitude and high frequency ( subthreshold oscillation ); or a maintain of his bistable activity for $\alpha<1.6$ when need is it. This last result is so important to neuron because is permit it to be efficient in his function to transmit information by a filtering of many process within before transmission to nervous message. 


\section{Data availability statements}

The data from simulations that support the findings of this study are available on request from the corresponding author, RY.

\section{Conflict of Interest}

The authors declare that they have no conflict of interest.

[1] A. N. Pisarchik and U. Feudel, Phys. Rep. 540, 167 (2014).

[2] A. Goldbeter, Biochemical Oscillations and Cellular Rythms. The molecular basis of periodic and chaotic behavior (Cambridge University Press, 1996).

[3] M. Alamgir and I. Epstein, J Am Chem Soc 105, 2500 (1983).

[4] Y. Loewenstein, S. Mahon, P. Chadderton, K. Kitamura, H. Sompolinsky, et al. Bistability of cerebellar Purkinje cells modulated by sensory stimulation. Nat Neurosci 8: 202-211 (2005).

[5] CC. Canavier, DA. Baxter, JW. Clark, JH. Byrne. Multiple modes of activity in a model neuron suggest a novel mechanism for the effects of neuromodulators. J. Neurophysiol. 72: 872-882. (1994)

[6] H. Lechner, D. Baxter, C. Clark, J. Byrne. Bistability and its regulation by serotonin in the endogenously bursting neuron R15 in Aplysia. J. Neurophysiol. 75: 957 (1996).

[7] I. Tagne Nkounga, F. M. Moukam Kakmeni and R. Yamapi, birhythmic oscillations and global stability analysis of a Conductance-based neuronal model under channel fluctuations, submitted in European physical journal plus, (2021)

[8] C. Moris, H. Lecar, Voltage oscillations in the barnacle giant muscle fiber, Biophys, J. 35, 193-213, (1981).

[9] R. Fitzhugh. Biophysical J.1,445,(1961).

[10] J. L. Hindmarsh, R. M. Rose., A model of the nerve impulse using two first oder differential equations, Nature Vol. 296,162-164,(1982).

[11] AL. Hodgkin, AF. Huxley, A Quantitative description of membrane current and its application to conduction and excitation in nerve, J physiol 117:500-544 (1952)

[12] B. Bao and Q. Yang, L. Zhu, H. Bao, Chaotic Bursting Dynamics and Coexisting Multistable Firing Patterns in 3D Autonomous Morris-Lecar Model and Microcontroller-Based Validations. International journal of bifurcation and chaos. Vol. 29, N 10, 1950134 (2019)

[13] R. Guttman, S. Lewis, J. Rinzel. Control of repetitive firing in squid axon membrane as a model for a neuroneoscillator. J Physiol 305: 377-395. (1980)

[14] P. Ghosh, S. Sen, S. Riaz, and D. S. Ray, Phys. Rev. E 83, 036205 (2011). 
[15] K. Pyragas, Phys. Lett. A 170, 421 (1995).

[16] J. L. Hindmarsh, R. M. Rose, A model of neuronal bursting using three coupled first order differential equations, Proc. Roy. Soc. London B221, 87-102 (1984).

[17] L. Kengne, J. Kengne, J. Roger, Coexisting bubbles, multiple attractors, and control of multistability in a simple Jerk system under the influence of a constant excitation force. J. Phys. 94:81 (2020)

[18] S. A. Prescott, Y. Koninck, T. J. Sejnowski. Biophysical basis for three distinct dynamical mechanisms of action potential initiation. PLoS Computational Biology, Vol. 4, Issue 11, (2008), p. 1000198.

[19] Z. Njitacke, T. Fonzin, C. Tchito, G. Dolvis, K. Wouapi and J. Kengne, Control of coexisting Attractors with Preselection of the Survived Attractor in Multistable Chua's System. Hindawi Complexity, Volume 2020, 5191085.

[20] G. Wang, F. Yuan, G. Chen, and Y. Zhang, Coexisting multiple attractors and riddler bassins of a memristive system. Entropy. 21, 678 (2019).

[21] K.Wouapi, B.Fotsin, F.Louodop, K.Feudjio, Z.Njitacke, T.Djeudjo, Various firing activities and finitetime synchronization of an improved Hindmarsh-Rose neuron model under electric field effect. Cogn Neurodyn, https://doi.org/10.1007/s11571-020-09570-0, Springer (2020).

[22] C. Yuan, Jiang Wang, Hopf bifurcation analysis and control of three-dimensional Prescott neuron model. Journal of Vibroengineering, Vol. 18, Issue 6 (2016)

[23] E. M. Izhikevich. Neural excitability, spiking and bursting, Int. J. Bifurcation and Chaos. (2000)

[24] J. Rinzel and Ermentrout, B. Analysis of neuralexcitability and oscillations, in Methods of Neural Modeling: From Synapses to Networks, eds. Koch, C.and Segev, I. (MIT Press) [1989].

[25] A. Venkatesan, M. Lakshmanan. Bifurcation and chaos in the double-well Duffing-Van der Pol oscillator: Numerical and analytical studies. Phys Rev E 1997; 6321:6330-56.

[26] P. Hagedorn. Non-linear oscillations. 2nd ed. Oxford: Clarendon Press; 1988.

[27] P. Cvitanovié, R. Artuso, R. Mainieri, G. Tanner and G. Vattay, CHAOS BOOK, Chaos- Book.org, version 13.3, (Sep 23 2010).

[28] AL. Shilnikov, G. Cymbaluyk. Coexistence of tonic spiking oscillations in a leech neuron model. Journal of Computational Neuroscience 18, 255-263 (2005).

[29] Rui Cai, Xiaoli Chen, Jinqiao Duan, Jürgen Kurths and Xianofan Li, Lévy noise-induced escape in an excitable system. J. Stat. Mech. 063503 (2017).

[30] N. F. Rulkov. Modeling of spiking-bursting neural behavior using two-dimensional map. Phys. Rev. E, 65, 2002

[31] J. L. Hindmarsh and R. M. Rose., Bvp models of nerve membrane (reply to c.ja game). Nature, 1982b299:375,(1982).

[32] A. H. Nayfeh, D. T. Mook, Nonlinear Oscillations (1979). 


\section{Appendix A}

$$
\begin{aligned}
& a=-g_{L}-0.5 g_{N a}+0.5 g_{N a} \frac{\beta_{m}+E_{N a}}{\gamma_{m}}-g_{N a} \frac{\beta_{m}^{3}}{6 \gamma_{m}^{3}}-0.5 g_{N a} E_{N a} \frac{\beta_{m}^{2}}{\gamma_{m}^{3}}+g_{N a} \frac{\beta_{m}^{5}}{15 \gamma_{m}^{5}} \\
&+g_{N a} E_{a} \frac{\beta_{m}^{4}}{3 \gamma_{m}^{5}}-8.5 g_{N a} \frac{\beta_{m}^{7}}{315 \gamma_{m}^{7}}-59.5 g_{N a} E_{N a} \frac{\beta_{m}^{6}}{315 \gamma_{m}^{7}} \\
& b= 0.5 g_{N a} \frac{1}{\gamma_{m}^{3}}+0.5 g_{N a} E_{N a} \frac{1}{3 \gamma_{m}^{3}}-2 g_{N a} \frac{\beta_{m}^{3}}{3 \gamma_{m}^{5}}-2 E_{N a} g_{N a} \frac{\beta_{m}^{2}}{3 \gamma_{m}^{5}}+59.5 g_{N a} \frac{\beta_{m}^{5}}{105 \gamma_{m}^{7}} \\
&+59.5 g_{N a} E_{N a} \frac{\beta_{m}^{4}}{63 \gamma_{m}^{7}} \\
& \theta_{1}=-g_{N a} \frac{\beta_{m}}{3 \gamma_{m}^{3}}+g_{N a} E_{N a} \frac{1}{15 \gamma_{m}^{5}}-59.5 g_{N a} \frac{\beta_{m}^{3}}{63 \gamma_{m}^{7}}-59.5 g_{N a} E_{N a} \frac{\beta_{m}^{2}}{63 \gamma_{m}^{7}}, \\
& \theta_{2}= 59.5 g_{N a} \frac{\beta_{m}}{315 \gamma_{m}^{7}}+8.5 g_{N a} E_{N a} \frac{1}{315 \gamma_{m}^{7}}, \\
& B=-g_{k} E_{k}, \\
& \alpha=-g_{a} E_{a}, \\
& \gamma_{1}= \frac{1}{6 \gamma_{w}}-\frac{\beta_{w}}{24 \gamma_{w}^{2}}-\frac{5 \beta_{w}^{2}}{16 \gamma_{w}^{3}}+\frac{11 \beta_{w}^{4}}{144 \gamma_{w}^{5}}-\frac{47 \beta_{w}^{6}}{1080 \gamma_{w}^{7}}-\frac{17 \beta_{w}^{8}}{1680 \gamma_{w}^{9}}, \\
& \gamma_{2}= \frac{1}{48 \gamma_{w}^{2}}+\frac{5 \beta_{w}}{16 \gamma_{w}^{3}}-\frac{11 \beta_{w}^{3}}{72 \gamma_{w}^{5}}+\frac{329 \beta_{w}^{5}}{2520 \gamma_{w}^{7}}+\frac{204 \beta_{w}^{7}}{5040 \gamma_{w}^{9}}, \\
& \gamma_{3}=-\frac{\beta_{w}^{2}}{24 \gamma_{w}^{2}}-\frac{1}{3}, \\
& \gamma_{4}= \frac{\beta_{z}^{2}}{48 \gamma_{z}^{2}}+\frac{5 \beta_{z}^{3}}{48 \gamma_{z}^{3}}-\frac{11 \beta_{z}^{5}}{720 \gamma_{z}^{5}}+\frac{47 \beta_{z}^{7}}{7560 \gamma_{z}^{7}}+\frac{17 \beta_{z}^{9}}{15120 \gamma_{z}^{9}}, \\
& c= \frac{\beta_{w}^{2}}{48 \gamma_{w}^{2}}+\frac{5 \beta_{w}^{3}}{48 \gamma_{w}^{3}}-\frac{11 \beta_{w}^{5}}{720 \gamma_{w}^{5}}+\frac{47 \beta_{w}^{7}}{7560 \gamma_{w}^{7}}+\frac{17 \beta_{w}^{9}}{15120 \gamma_{w}^{9}}, \\
& r= \frac{\beta_{z}^{2}}{24 \gamma_{z}^{2}}+\frac{1}{3}, \\
& s\left[\frac{1}{6 \gamma_{w}}-\frac{\beta_{w}}{24 \gamma_{w}^{2}}-\frac{5 \beta_{w}^{2}}{16 \gamma_{w}^{3}}+\frac{11 \beta_{w}^{4}}{144 \gamma_{w}^{5}}-\frac{47 \beta_{w}^{6}}{1080 \gamma_{w}^{7}}-\frac{17 \beta_{w}^{8}}{1680 \gamma_{w}^{9}}\right] \\
&= \\
&=
\end{aligned}
$$

The above constants can be estimated if we refer to the parameters of the improved ML neuronal model defined in [12], for example with $C=1, g_{L}=2.0, E_{L}=-70.0, g_{N a}=20.0, E_{N a}=50.0, g_{K}=20.0, E_{K}=$ $-100.0, g_{a}=2.0, E_{a}=-100.0, \beta_{m}=-1.2, \gamma_{m}=18.0, \beta_{w}=-20.0, \gamma_{w}=15.0, \beta_{z}=-21.0, \gamma_{z}=10.0$ and $\phi_{w}=\phi_{z}=0.15$, we can say that these constants will be of the order: $0<a \leq 2.85 ; 0<b \leq 236$; $0<\theta_{1} \leq 989 ; 0<\theta_{2} \leq 1.76 \times 10^{4} ; 0<B \leq 189.19 ; 0<\gamma_{1} \leq 106.5 ; 0<\alpha \leq-200.00<\gamma_{2} \leq-0.3$; $0<s \leq-213 ; 0<r \leq 0.45 ; 0 \leq \gamma_{4} \leq \ldots$. and $0 \leq c \leq \ldots \ldots$ 